\title{
IFI16 promotes human embryonic stem cell trilineage specification through interaction with p53
}

\author{
Qian $\mathrm{He} \mathbb{D}^{1,2,3,4}$, Zubiao $\mathrm{Wu}^{1,3,4}$, Wei Yang ${ }^{1,3,4}$, Doukou Jiang ${ }^{1,3,4}$, Chaofeng $\mathrm{Hu}^{2}$, Xiaofei Yang $\mathbb{D}^{1,3,4,5 凶}, \mathrm{Ning} \mathrm{Li} \mathbb{D}^{1,3,4,5 凶}$ and \\ Furong $\mathrm{Li}\left(\mathbb{D}^{1,3,4,5 凶}\right.$
}

Transcriptional regulation plays an essential role in the self-renewal and differentiation of human embryonic stem cells (hESCs). However, how external signals disrupt the self-renewal regulatory network and further drive hESC differentiation remains largely unknown. Here, we found the immune regulative protein, gamma-interferon-inducible protein 16 (IFI16) was involved in the regulation of both self-renewal and differentiation gene expression during hESC trilineage specification through interaction with p53. IFI16 expression levels were upregulated through JNK activation. IFI16 knockdown delayed the downregulation of self-renewal gene expression and suppressed the upregulation of differentiation gene expression, while IFI16 overexpression accelerated trilineage specification. Furthermore, IFI16 stabilized p53-binding in the genome through IFI16-p53 interaction and differentially regulated self-renewal and differentiation gene expression. Together, our results suggest a particular role of IFI16 in differential gene expression regulation during trilineage specification of hESCs in a manner that is dependent on the genome-wide profile of p53-binding directed by IFI16-p53 interaction.

npj Regenerative Medicine (2020)5:18; https://doi.org/10.1038/s41536-020-00104-0

\section{INTRODUCTION}

Although human embryonic stem cells (hESCs) and somatic cells share almost identical DNA sequence information, hESCs maintain pluripotency through selective gene expression. According to the protocol proposed by Shinya Yamanaka, somatic cells can be reprogrammed into pluripotent stem cells by ectopic expression of transcription factors such as OCT4 and SOX $2^{1}$, suggesting that transcriptional regulation plays a key role in the pluripotency maintenance of hESCs. Indeed, OCT4, SOX2, and Nanog form a core self-renewal regulatory network to maintain the pluripotent state of $\mathrm{hESCs} \mathrm{s}^{2,3}$. The cooperation of these transcription factor regulators, epigenetic modifiers and effectors of external signaling pathways maintain the pluripotency of hESCs${ }^{2}$. Although the transcriptional regulatory network of the pluripotent state in hESCs has been described, how external signals disrupt the core self-renewal regulatory network and further drive the differentiation of hESCs remains largely unclear.

Gamma interferon inducible protein 16 (IFI16), a member of p200 family, was first identified in lymphoid cells and was considered as an intracellular DNA sensor in the regulation of the immune responses ${ }^{4-6}$. Beyond that, evidence supports that IFI16 contributes to genome remodeling in virus defense through interaction with histone $\mathrm{H} 2 \mathrm{~B}$ or $\mathrm{H} 3 \mathrm{~K} 9$ methyltransferase ${ }^{7,8}$. Moreover, an observation that IFI16 upregulation induced by LIF mediating the cell cycle arrest has been demonstrated in medullary thyroid carcinoma cells ${ }^{9}$. Therefore, those findings above demonstrate a potential role of IFI16 in cell proliferation and nuclear protein binding. However, in addition to immunoregulation, whether IFI16 participates in other physiological process especially in self-renewal and differentiation of hESCs is still unknown.
As one of the most important tumor suppressors, p53 functions in cell cycle arrest and apoptosis during aberrant oncogene activation and senescence of somatic cells after genomic instability ${ }^{10,11}$. Beyond that, p53 also plays an important role in the self-renewal and differentiation of ESCs ${ }^{12}$. p53 expression level has been upregulated during hESCs early differentiation and p53 knockdown reduces the spontaneous differentiation ${ }^{13}$. In addition, p53 suppresses Nanog expression and sufficiently induces mouse embryonic stem cells (mESCs) differentiation ${ }^{14}$, and OCT4 maintains the pluripotency by inactivating $\mathrm{p} 53$ in $\mathrm{hESCs}^{15}$. Furthermore, p53 regulates LncPRESS1 and coordinates Wnt/Nodal signals contributing to hESCs differentiation ${ }^{16,17}$. However, activation of a transcription factor would result in a spectrum of gene expression change and further affect the downstream signals ${ }^{18,19}$. Despite an extensive understanding of $\mathrm{p} 53$ function in regulation of the self-renewal and differentiation of ESCs, the genome-wide profiling of p53 targets and corresponding gene expression during hESCs differentiation is still largely unknown. Remarkably, the crystal structures of both HIN-A and HIN-B domains of IFI16 interact with $\mathrm{p} 53^{20}$. Therefore, it is promising to identify the IFI16/ p53 interaction endogenously and explore whether IFI16/ p53 signal could directly affect hESCs differentiation.

In this study, we aimed to investigate whether IFI16 would participate in the regulation of hESCs differentiation. We report that JNK activation induced IFI16 upregulation which stabilized p53-binding in the genome through IFI16-p53 interaction and further differentially regulated self-renewal and differentiation gene expression to determine the trilineage specification of hESCs.

\footnotetext{
${ }^{1}$ Translational Medicine Collaborative Innovation Center, The Second Clinical Medical College (Shenzhen People's Hospital), Jinan University, 518020 Shenzhen, China. ${ }^{2}$ Integrated Chinese and Western Medicine Postdoctoral Research Station, Jinan University, 510632 Guangzhou, China. ${ }^{3}$ Guangdong Engineering Technology Research Center of Stem Cell and Cell therapy, 518020 Shenzhen, China. ${ }^{4}$ Shenzhen Key Laboratory of Stem Cell Research and Clinical Transformation, 518020 Shenzhen, China. ${ }^{5}$ Present address: Translational Medicine Collaborative Innovation Center, The Second Clinical Medical College (Shenzhen People's Hospital), Jinan University, 1017 Dongmen North Road, 518020 Shenzhen, China. ${ }^{\circledR}$ email: sophiayangxf@163.com; lining.yatu@hotmail.com; frli62@163.com
} 


\section{RESULTS}

IFI16 is specifically upregulated during trilineage specification To investigate the role of gamma-interferon-inducible protein 16 (IFI16) in the self-renewal and differentiation of human embryonic stem cells (hESCs), we used hESC line H9 to derive embryonic stages of endoderm, mesoderm and ectoderm. Initially we measure the IFI16 expression levels in hESCs and trilineage differentiated cells, and found that the IFI16 mRNA and protein levels were gradually upregulated during the trilineage specification. As shown in Fig. 1a-f and Supplementary Fig. 1d-f, a low expression level of IFI16 was observed in H9 cells, while IFI16 expression reached a medium level at Day 2 of trilineage specification and had a huge potentiation in the final stages (Day 5 of endoderm/mesoderm and Day 7 of ectoderm). To verify the specificity of IFI16 regulation during trilineage specification, we examined the expression levels of another two intracellular DNA sensor, Absent In Melanoma 2 (AIM2) and cyclic GMP-AMP Synthase (cGAS). In contrast, the mRNA and protein expression levels of AIM2 were not significantly changed during the trilineage specification (Fig. 1d-f and Supplementary Fig. 1a-f). In addition, the expression level of cGAS was not changed during the endoderm specification, while remarkably downregulated when differentiating into the mesoderm and ectoderm (Fig. 1d-f and Supplementary Fig. 1a-c and g-i). To confirm the IFI16 expression during the trilineage specification, the IFI16 protein were in situ stained through immunofluorescence. Consistent with the results from western blots, low IFI16 expression was evident in $\mathrm{H} 9$ cells, while IFI16 expression levels were greatly upregulated in the nuclei of endoderm, mesoderm and ectoderm cells (Fig. 1g). Meanwhile, AIM2 had a relatively stable expression during the trilineage specification (Fig. 1g), and the expression level of cGAS was reduced during the mesoderm and ectoderm specification, while no significant change was observed during the endoderm specification (Fig. 1g). Together, these results provide the initial evidence that IFI16 protein levels are specifically upregulated during the trilineage specification.

\section{JNK activation mediates IFI16 upregulation during trilineage specification}

We next explored the possible mechanism underlying the upregulation of IFI16 expression. The observation from quantitative real-time PCR (qPCR) results (Fig. 1a-c) suggested that the upregulation of IFI16 expression was probably attributed to its transcriptional activation. Given that the mitogen-activated protein kinase (MAPK) plays an important role in the regulation of cellular proliferation and differentiation ${ }^{21-23}$, we assessed the activities of MAPK families including extracellular signal-regulated kinases (ERKs, p42, and p44), the c-Jun N-terminal kinases (JNKs) and the p38 MAP kinases. As shown in Fig. 2a-c, the ERK, JNK, and p38 kinases were all activated within $4 \mathrm{~h}$ after the trilineage specification as evidenced by their respective phosphorylations. To investigate the role of MAPKs in the upregulation of IFI16, we further examined the IFI16 mRNA levels in the trilineage cells after inhibition of these kinases. Incubating H9 cells with SP600125, U0126, or SB203580, the specific inhibitors of JNK, ERK, or p38 MAP kinases respectively, did not change the IFI16 mRNA levels (Fig. 2d-f). However, the upregulation of IFI16 during the trilineage specification was greatly inhibited by SP600125 (Fig. $2 d)$. In contrast, no significant changes were observed in IFI16 mRNA expression after U0126 or SB203580 incubation (Fig. 2e, f). Since c-Jun has a validated binding motif in IFI16 promoter ${ }^{24}$, we then performed chromosome immunoprecipitation (ChIP) to evaluate the transcriptional activity of IFI16 promoter. We found that the IFI16 promoter was remarkably enriched during the trilineage specification by c-Jun antibodies, while the binding levels of IFI16 3' untranslated regions (UTR), AIM2 and CGAS promoter were not affected (Fig. 2g, h). Taken together, these results suggest that JNK pathway specifically participates in the transcriptional activation of IFI16.

\section{IFI16 knockdown inhibits trilineage specification}

To further investigate the role of IFI16 in the self-renewal and differentiation of hESCs, we knocked down IFI16 by engineering the lentivirus in which expression of two short hairpin RNAs (shRNAs), sh1865 or sh2153. Due to the relatively low expression, sh1865 or sh2153 did not significantly change IFI16 expression in H9 cells (Supplementary Fig. 2a-d). However, IFI16 protein levels after the trilineage specification were greatly reduced by sh1865 and sh2153 (Supplementary Fig. 2a-d). Meanwhile, IFI16 knockdown did not affect the cell viability measured by the CCK- 8 assays in hESCs or during the trilineage specification (Supplementary Fig. 2e-h). Notably, by qPCR and western blot analysis, we found that sh1865 and sh2153 remarkably suppressed the downregulation of the self-renewal transcriptional factors including OCT4, SOX2, and KLF4 during the trilineage specification (Fig. 3a-f, Supplementary Fig. 3a-c, Supplementary Fig. 4a-f and Supplementary Fig. $5 \mathrm{a}-\mathrm{c}$ ). Moreover, the upregulation of trilineage marker genes including SOX $17^{25}$, FOXA2 ${ }^{26}$ and $C X C R 4^{27}$ for endoderm, Brachyury ${ }^{28}$ and CXCR4 ${ }^{29}$ for mesoderm, and PAX $6^{30}$, OTX $2^{31}$ for ectoderm, were significantly reduced by sh1865 and sh2153 (Fig. 3a-f, Supplementary Fig. 3a-c, Supplementary Fig. $4 a-f$ and Supplementary Fig. 5a-c).

To further validate the role IFI16 in regulation of the selfrenewal and differentiation gene expression, we performed flow cytometry and immunofluorescence. The SOX $17^{+} / \mathrm{FOXA2} 2^{+}$population was referred to as endoderm cells and Brachyury ${ }^{+} / \mathrm{CXCR} 4^{+}$, $\mathrm{PAX6}^{+} /$Nestin $^{+32}$ were referred to as mesoderm and ectoderm cells, respectively. The endoderm, mesoderm and ectoderm population were all remarkably decreased by sh1865 and sh2153 (Fig. 3g, Supplementary Fig. 3d-f, Supplementary Fig. 4g and Supplementary Fig. 5d-f). Moreover, the fluorescence intensity of OCT4 and SOX2 was significantly upregulated by sh1865 and sh2153 during the trilineage specification, while trilineage marker genes including SOX17, FOXA2, Brachyury, SNAI2 ${ }^{33}$ and PAX6 were significantly reduced by sh1865 and sh2153 (Fig. 3h, Supplementary Fig. 3g-i, Supplementary Fig. 4h and Supplementary Fig. 5g-i). Collectively, our results suggest that IFI16 knockdown delays the downregulation of self-renewal gene expression and suppresses the upregulation of differentiation gene expression.

\section{IFI16 overexpression accelerates trilineage specification}

To provide further evidence that regulation of hESCs self-renewal and differentiation by IFI16, we generated a lentiviral construct encoding the IFI16 gene that can be induced by doxycycline (DOX). As shown in Supplementary Fig. 6a, b, after infection, DOX induced a time course dependent exogenesis expression of IFI16 in H9 cells. Notably, DOX-induced IFI16 expression did not significantly change OCT4 and SOX2 protein levels in H9 cells (Supplementary Fig. 6c, d). In addition, alkaline phosphatase (AP) staining showed that there was no significant difference among the AP positive proliferative colonies in DOX-induced IFI16 expression group, no-DOX induction group and negative control group (Supplementary Fig. 6e). These results indicate that IFI16 overexpression alone does not affect the self-renewal ability of the hESCs.

In the next attempt to study whether IFI16 overexpression regulates hESCs differentiation, we measured the differentiation gene expression. Results from western blots showed that the expression of trilineage marker genes including SOX17, FOXA2, Brachyury, SNAI2, and PAX6 were not affected by DOXinduced IFI16 expression in the final stages of trilineage induction (Supplementary Fig. 7a-f). Moreover, as shown in Supplementary Fig. $7 \mathrm{~g}-\mathrm{i}$, the endoderm, mesoderm, and 
a

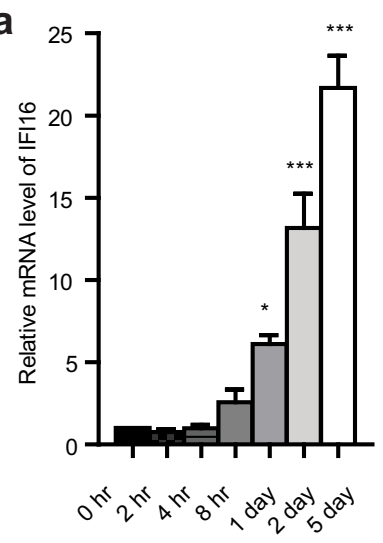

b

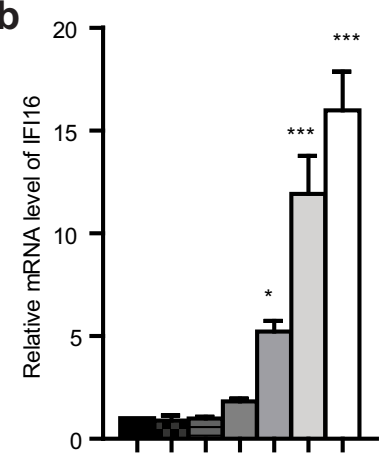

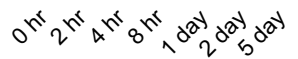

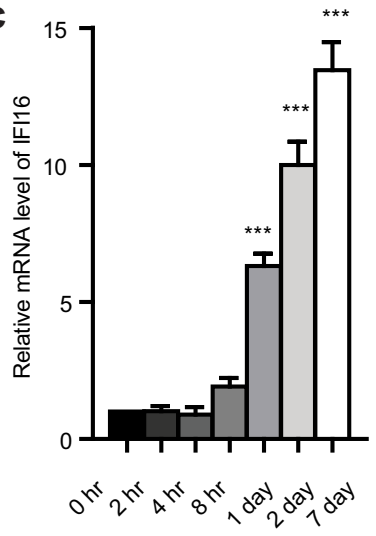

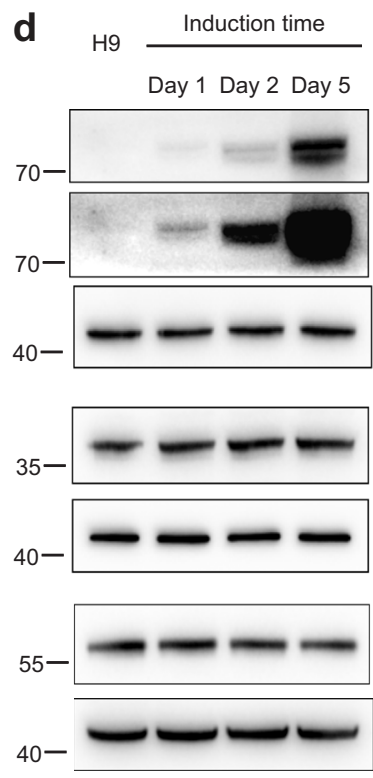

e
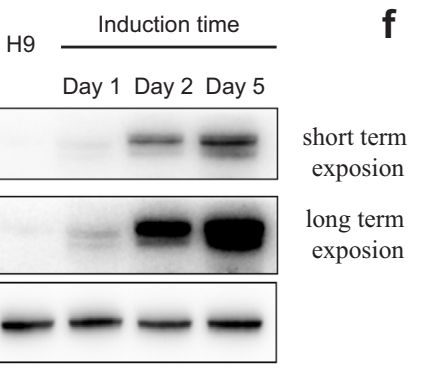

H9 $\frac{\text { Induction time }}{\text { Day } 1 \text { Day } 2 \text { Day } 7}$
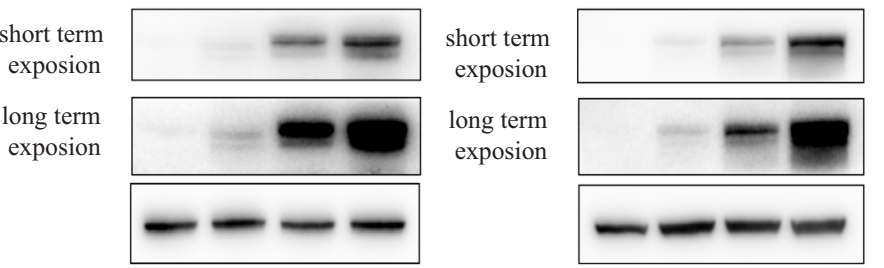

short term exposion

long term exposion

$\beta$-actin

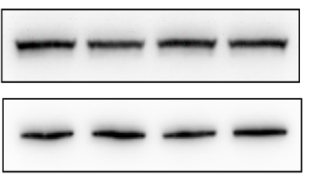

AIM2
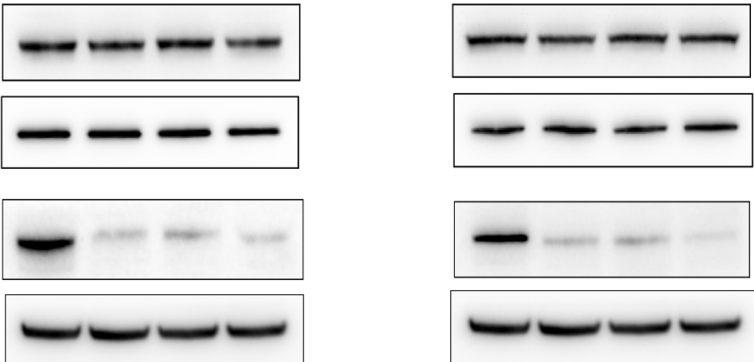

cGAS

$\beta$-actin
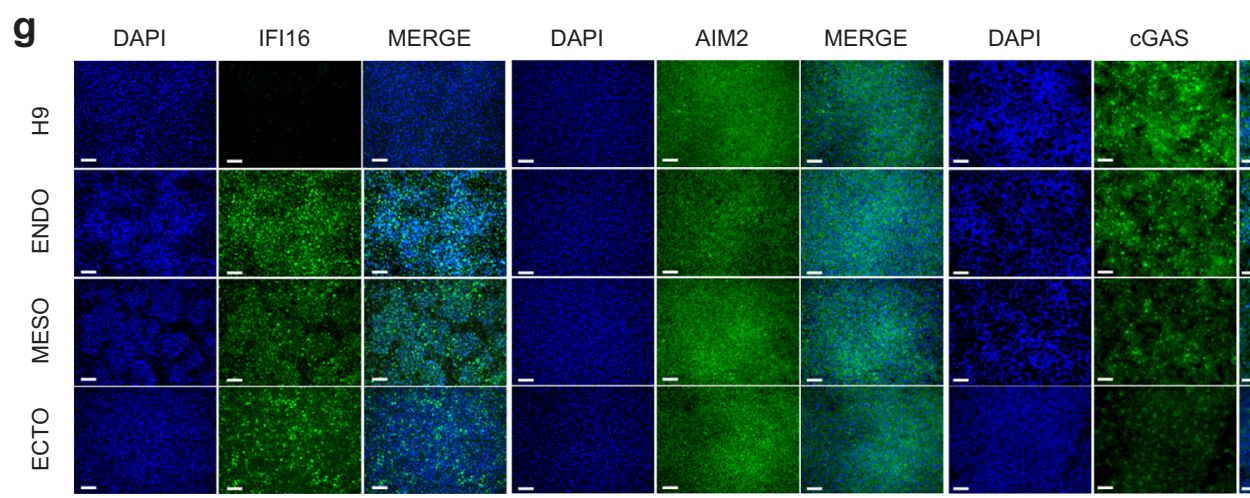

MERGE

Fig. 1 Upregulation of IFI16 expression during trilineage specification. a-c Quantitative PCR analysis of IFI16 mRNA levels in H9 cells and differentiated trilineage ( $\mathbf{a}$, endoderm; $\mathbf{b}$, mesoderm; $\mathbf{c}$, ectoderm; $n=4$ in each group) for indicated periods of time. The relative mRNA levels of IFI16 in indicated time courses were calculated relatively to which at 0 h. $\mathbf{d}$-f Representative immunoblots of IFI16, AIM2, and cGAS from H9 cells and differentiated trilineage (d, endoderm; e, mesoderm; f, ectoderm) for indicated periods of time. $\beta$-actin serves as a loading control. g Representative immunofluorescence images staining with antibodies against IFI16, AIM2, and cGAS in H9 cells and the differentiated trilineage. DAPI serves as a nucleus indicator. Scale bar, $200 \mu \mathrm{M}$. ENDO, endoderm; MESO, mesoderm; ECTO, ectoderm. All data were presented as mean \pm SEM. Comparisons between groups for statistical significance were performed with one-way ANOVA with Tukey's post hoc test. ${ }^{*} P<0.01,{ }^{* * *} P<0.001$ versus $\mathrm{H} 9$.

ectoderm population in FACS were not changed either. Due to the relatively high expression levels of IFI16 in the final stages of trilineage induction (Fig. 1), we thereafter hypothesized that IFI16 overexpression would be involved in the regulation of the
hESCs self-renewal and differentiation in the early stage of trilineage induction. Indeed, on Day 2 of trilineage induction, the downregulation of OCT4 and SOX2 levels were further enhanced by IFI16 expression according to the GPCR and 


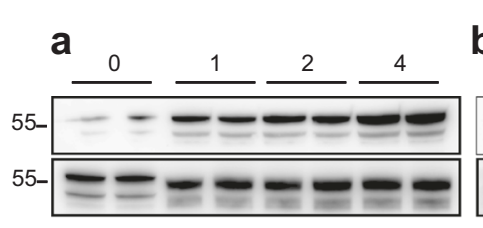

b

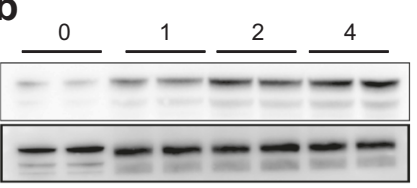

C

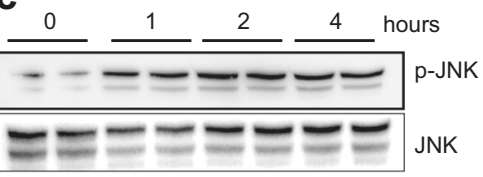

40_
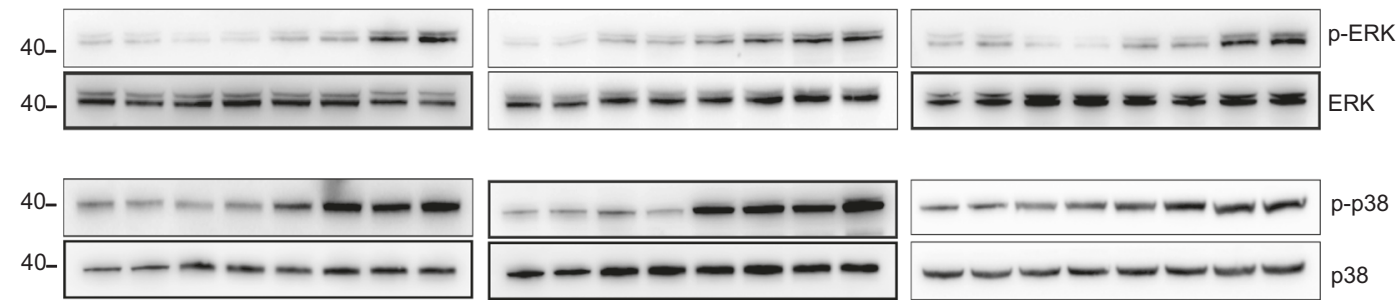

d

NC

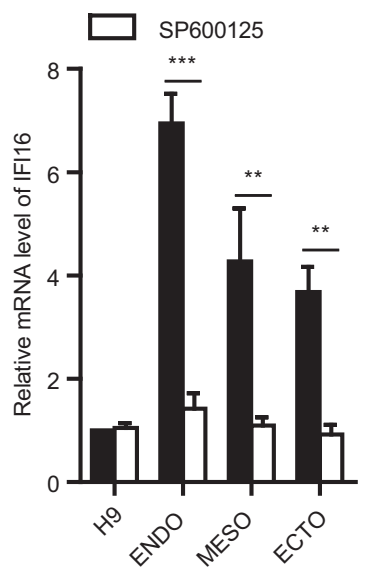

e
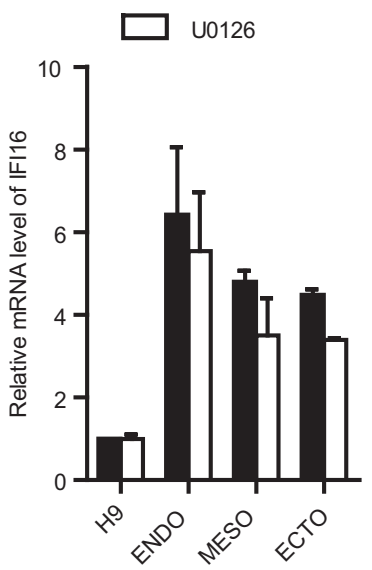

f
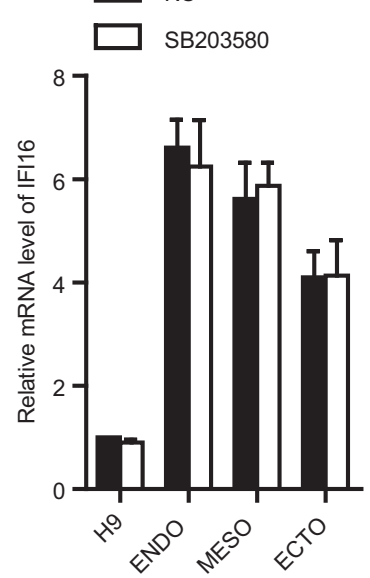

g
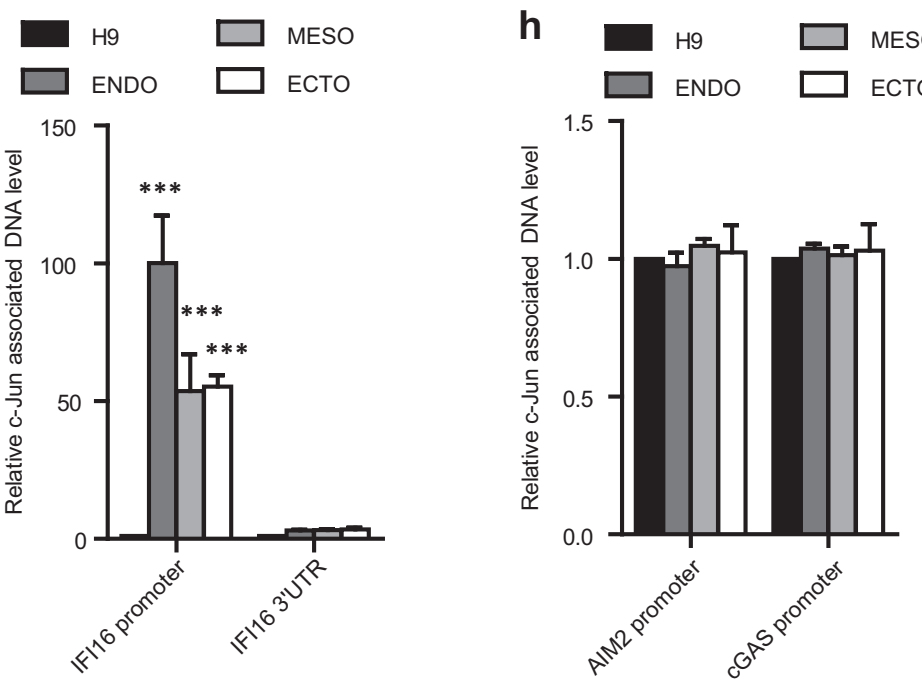

Fig. 2 JNK activation is responsible for IFI16 upregulation during trilineage specification. a-c Representative immunoblots of total lysates from H9 cells and differentiated trilineage ( $\mathbf{a}$, endoderm; $\mathbf{b}$, mesoderm; $\mathbf{c}$, ectoderm) for indicated periods of time and probed with the antibodies for p-JNK, JNK, p-ERK, ERK, for p-p38 and p38. d-f, quantitative PCR analysis of IFI16 mRNA levels in H9 cells and differentiated trilineage after incubation with SP600125 $(10 \mu \mathrm{M}, n=4,(\mathbf{d}), \mathrm{U} 0126(10 \mu \mathrm{M}, n=3,(\mathbf{e})$, and SB203580 $(10 \mu \mathrm{M}, n=4$, f The relative mRNA level of IFI16 was calculated relatively to which in H9 cells. $\mathbf{g}$, $\mathbf{h}$ quantitative PCR examination of IFI16 promoter $(n=4)$, IFI16 3'UTR $(n=4)$, AIM2 promoter $(n=3)$, and cGAS promoter $(n=3)$ levels pulled-down by c-Jun antibodies in $\mathrm{H} 9$ cells and differentiated trilineage. The relative c-Jun associated DNA level was calculated relatively to which in $\mathrm{H} 9$ cells. ENDO, endoderm; MESO, mesoderm; ECTO, ectoderm. NC, negative control. All data were presented as mean \pm SEM. Comparisons between groups for statistical significance were performed with one-way ANOVA with Tukey's post hoc test $(\mathbf{g}, \mathbf{h})$ or two-way ANOVA with Bonferroni post hoc test $(\mathbf{d}-\mathbf{f}) .{ }^{*} P<0.05,{ }^{* *} P<0.01,{ }^{* * *} P<0.001$ versus 0 h, NC or H9. 


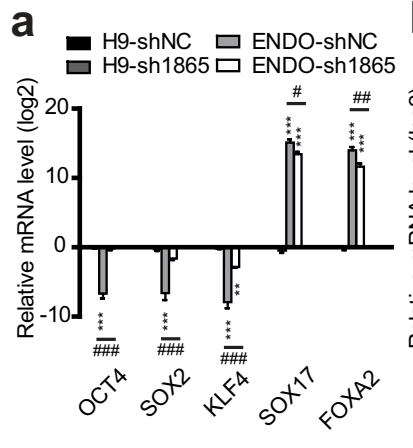

b

b H9-shNC 口MESO-shNC C 口 H9-sh1865 ㅁ MESO-sh1865

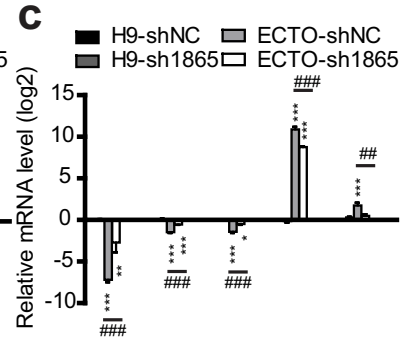

\#\#册 \#

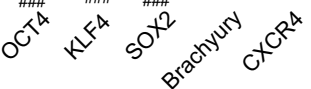
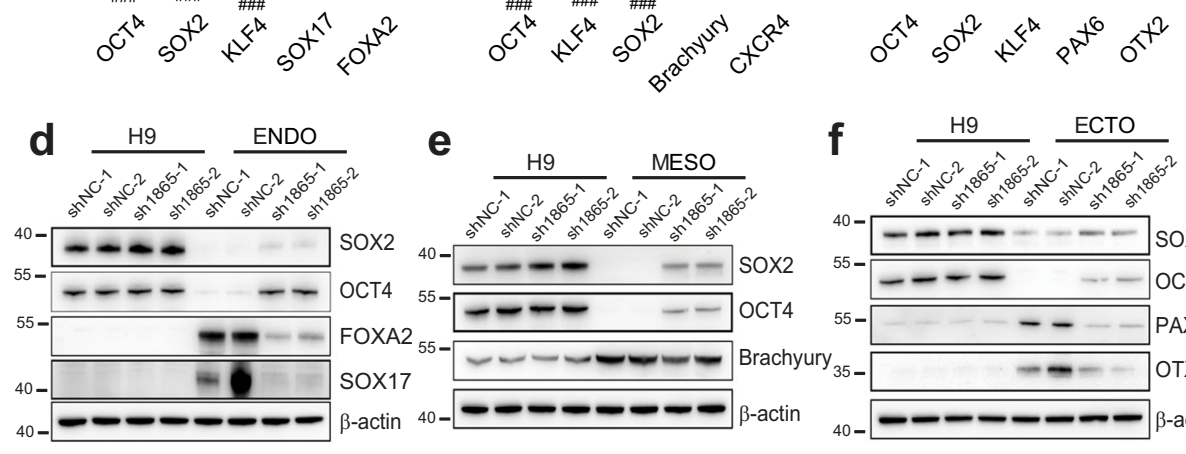

e
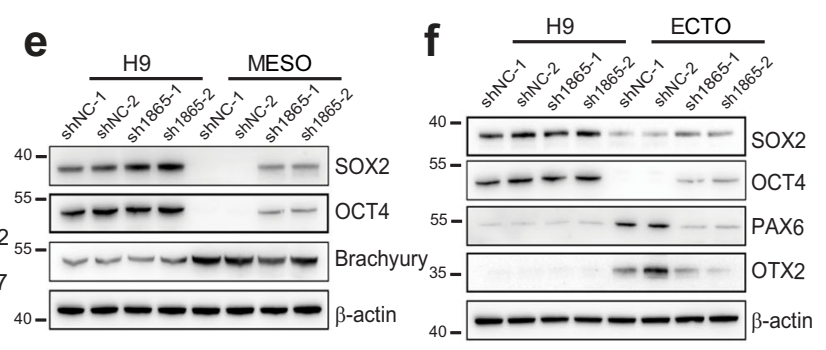

sh1865
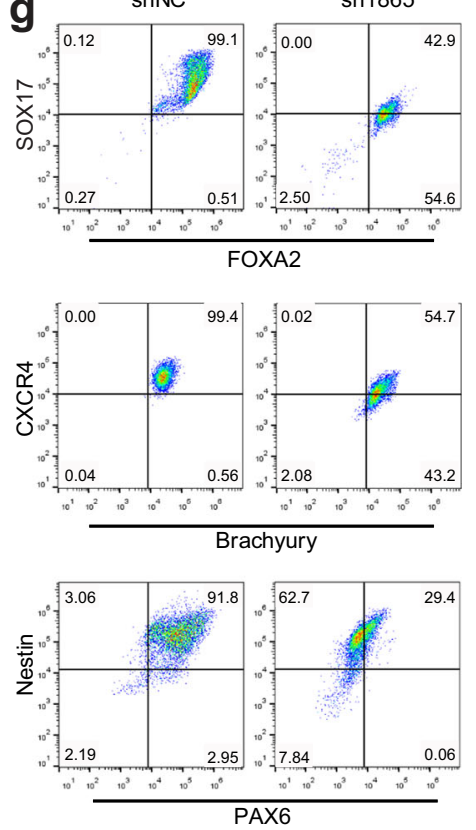

h
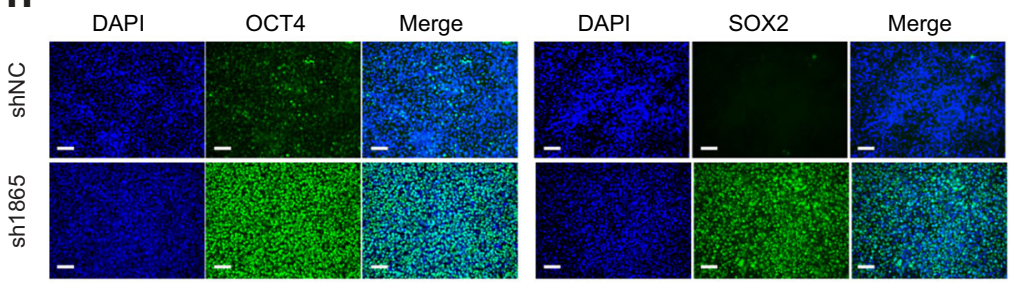

DAPI

SOX17

FOXA2
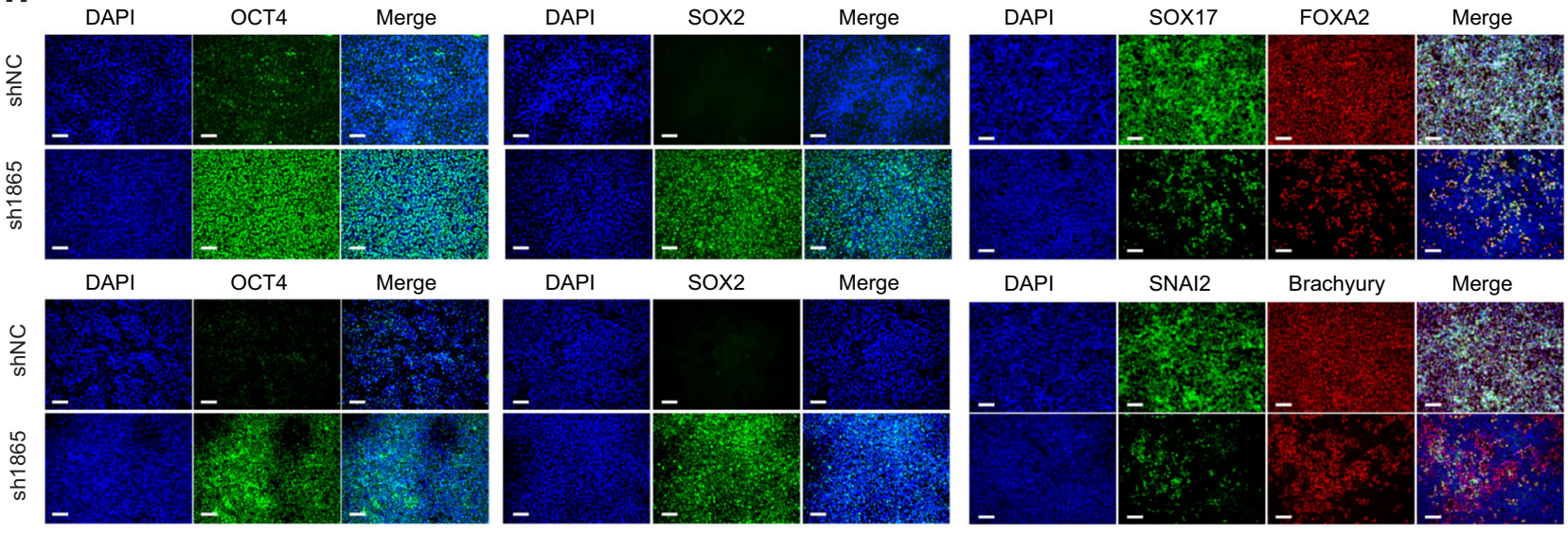

DAPI

sox2

Merge
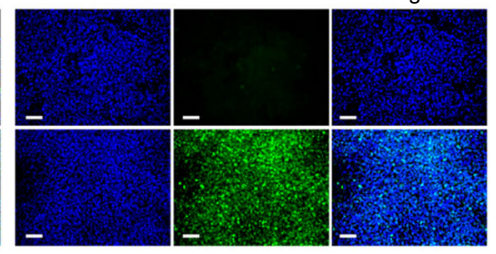

DAPI

SNAI2

Brachyury

Merge
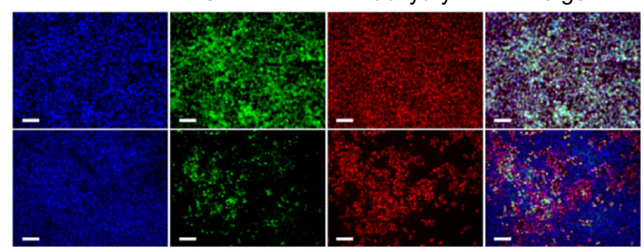

DAPI

SOX2

Merge
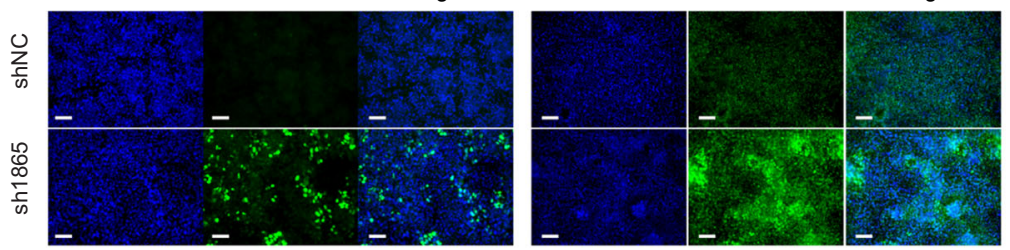

DAPI

PAX6

Merge

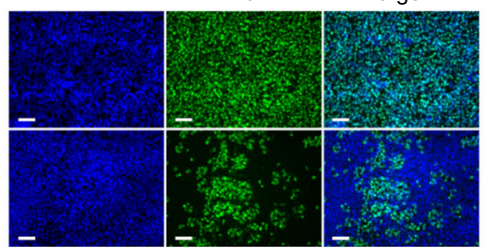

Fig. 3 IFI16 knockdown by sh1865 inhibits trilineage specification. a-c Quantitative PCR examination of OCT4, SOX2, KLF4, SOX17, FOXA2, CXCR4, Brachyury, PAX6, and OTX2 mRNA levels in H9 cells and differentiated trilineage (a, endoderm; $\mathbf{b}$, mesoderm; $\mathbf{c}$, ectoderm; $n=4$ in each group) infected with sh1865 or shNC. The relative mRNA level of each gene was calculated relatively to which in H9-shNC group. d-f Representative immunoblots of total lysates from $\mathrm{H} 9$ cells and differentiated trilineage (d, endoderm; e, mesoderm; $\mathbf{f}$, ectoderm) infected with sh1865 or shNC and probed with the antibodies for OCT4, SOX2, SOX17, FOXA2, Brachyury, PAX6, and OTX2. $\beta$-actin serves as a loading control. g Flow cytometric analysis of SOX $17^{+} / \mathrm{FOXA}^{+}$, Brachyury ${ }^{+} / \mathrm{CXCR} 4^{+}$, and $\mathrm{PAX}^{+} / \mathrm{Nestin}^{+}$population in differentiated trilineage infected with sh1865 or shNC, The signals in the fourth quadrant indicate endoderm, mesoderm, or ectoderm population. The number in each quadrant means the proportion in total cell population. $\mathbf{h}$ Representative immunofluorescence images staining with antibodies against OCT4, SOX2, SOX17, FOXA2, Brachyury, SNAI2, and PAX6 in the differentiated trilineage infected with sh1865 or shNC. Upper rows, endoderm; middle rows, mesoderm; bottom rows, ectoderm. DAPI serves as a nucleus indicator. Scale bar, $200 \mu M$. ENDO, endoderm; MESO, mesoderm; ECTO, ectoderm. NC, negative control. All data were presented as mean \pm SEM. Comparisons between groups for statistical significance were performed with one-way ANOVA with Tukey's post hoc test $(\mathbf{a}-\mathbf{c}) .{ }^{*} P<0.05,{ }^{* *} P<0.01,{ }^{* *} P<0.001$ versus H9-NC. ${ }^{\#} P<0.05$, ${ }^{\# \#} P<0.01$, $\# \#$ \# 0.001 versus H9-ENDO, H9-MESO, or H9-ECTO.

western blot analysis (Fig. 4a-f and Supplementary Fig. 8a-c). In addition, the upregulation of trilineage marker genes including SOX17, FOXA2, Brachyury, OTX2, and PAX6 were also potentiated (Fig. 4a-f and Supplementary Fig. $8 a-c$ ). Moreover, the endoderm and ectoderm population in FACS were greatly enlarged by IFI16 expression, while the mesoderm population had a moderate change (Fig. $4 \mathrm{~g}$ and Supplementary Fig. 8d-f). Furthermore, the fluorescence intensity of OCT4 and SOX2 was 

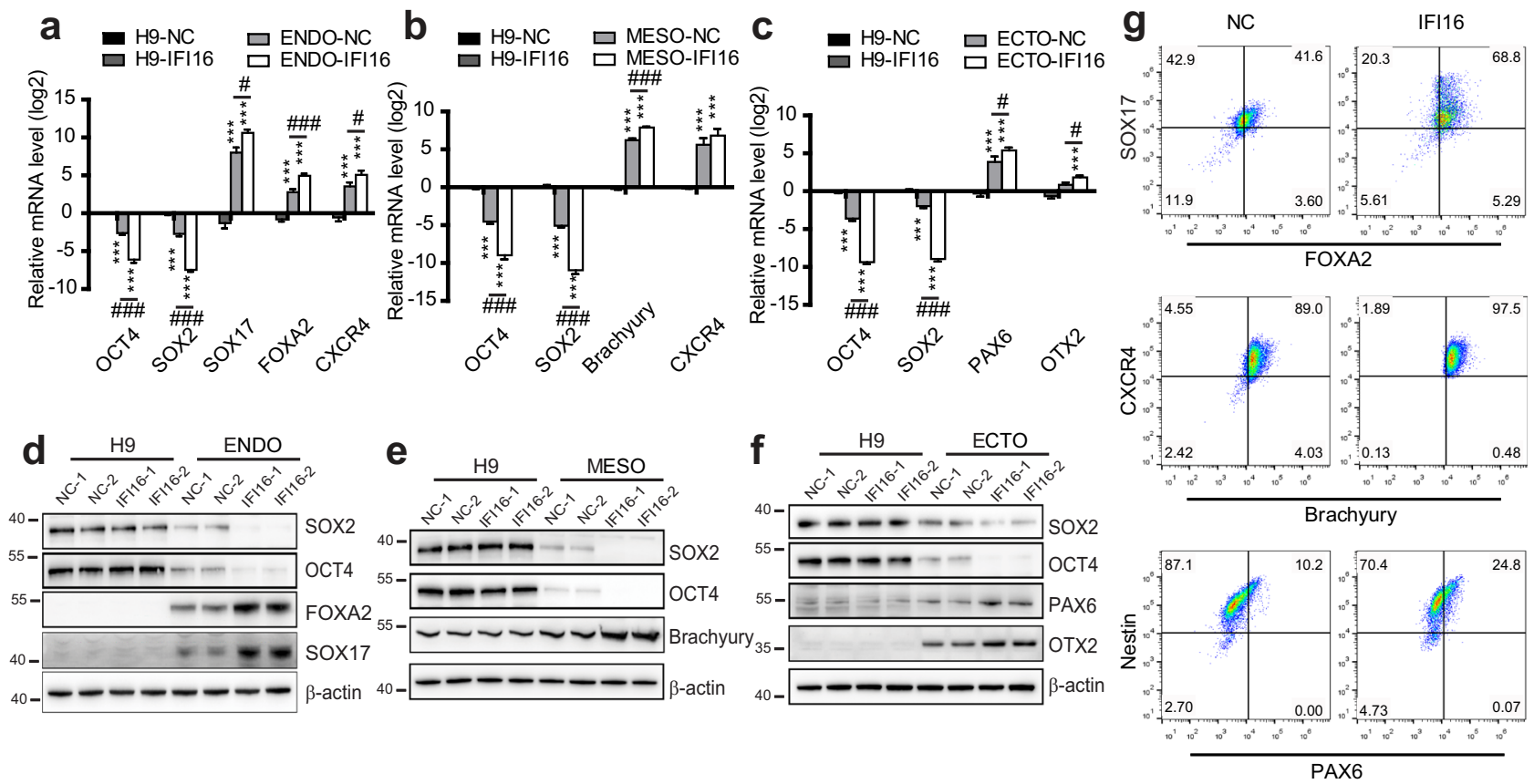

h

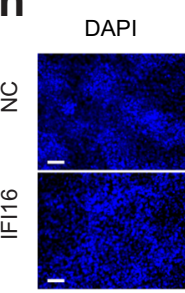

OCT4

Merge

DAPI

sox2

Merge

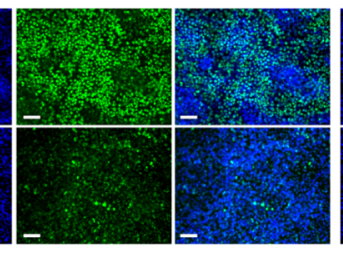

DAPI

OCT4

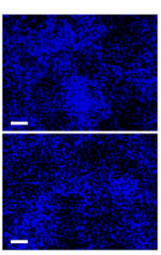

DAPI

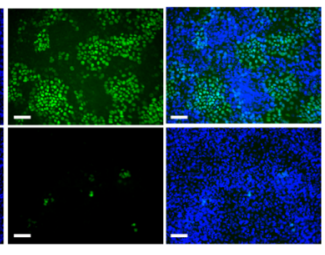

SOX2

Merge
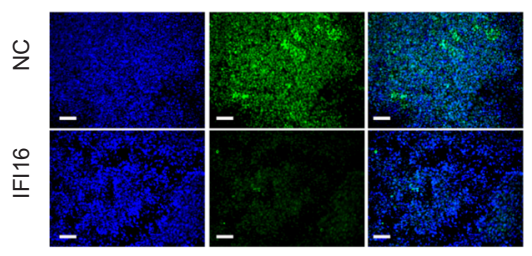

DAPI

OCT4

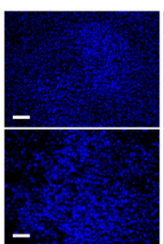

DAPI
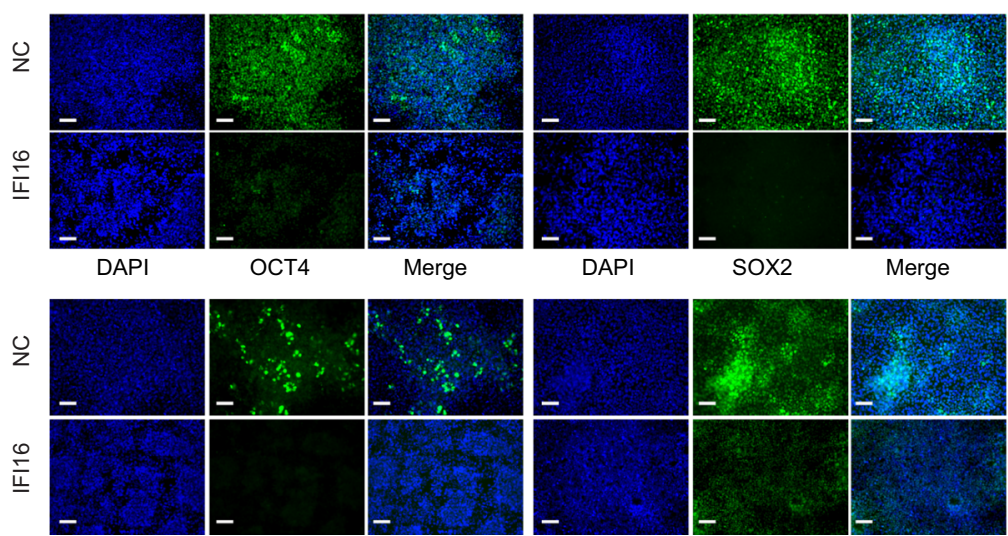

sox2

Merge

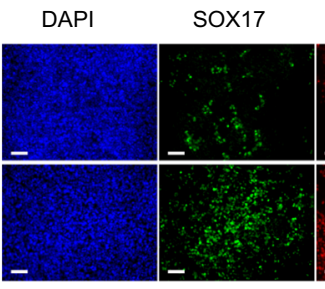

FOXA2

Merge
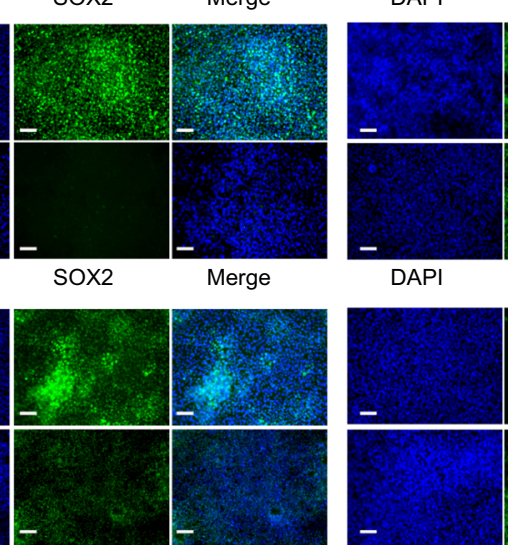

SNAI2

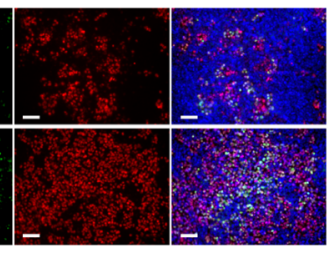

Brachyury

Merge

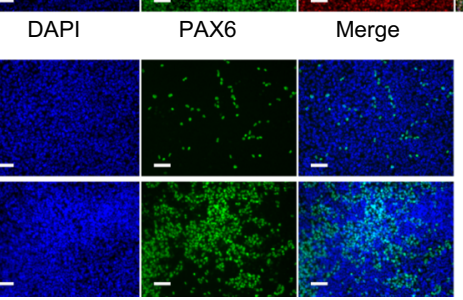

Fig. 4 IFI16 overexpression accelerates trilineage specification. a-c Quantitative PCR analysis of OCT4, SOX2, SOX17, FOXA2, CXCR4, Brachyury, PAX6, and OTX2 mRNA levels in H9 cells and differentiated trilineage on Day 2 ( $\mathbf{a}$, endoderm; $\mathbf{b}$, mesoderm; $\mathbf{c}$, ectoderm; $n=4$ in each group) infected with IFI16 or NC. The relative mRNA level of each gene was calculated relatively to which in H9-NC group. d-f Representative immunoblots of total lysates from H9 cells and differentiated trilineage on Day 2 (d, endoderm; e, mesoderm; $\mathbf{f}$, ectoderm) infected with IFI16 or NC and probed with the antibodies for OCT4, SOX2, SOX17, FOXA2, Brachyury, PAX6, and OTX2. $\beta$-actin serves as a loading control. g Flow cytometric analysis of SOX $17^{+} /$FOXA $^{+}$, Brachyury ${ }^{+} / \mathrm{CXCR}^{+}$, and PAX $6^{+} /$Nestin $^{+}$population in differentiated trilineage on Day 2 infected with IFI16 or NC, The signals in the fourth quadrant indicate endoderm, mesoderm, or ectoderm population. The number in each quadrant means the proportion in total cell population. $\mathbf{h}$ Representative immunofluorescence images staining with antibodies against OCT4, SOX2, SOX17, FOXA2, Brachyury, SNAI2, and PAX6 in the differentiated trilineage on Day 2 infected with IFI16 or NC. Upper rows, endoderm; middle rows, mesoderm; bottom rows, ectoderm. DAPI serves as a nucleus indicator. Scale bar, $200 \mu M$. ENDO, endoderm; MESO, mesoderm; ECTO, ectoderm. NC, negative control. All data were presented as mean \pm SEM. Comparisons between groups for statistical significance were performed with one-way ANOVA with Tukey's post hoc test $(\mathbf{a}-\mathbf{c}) .{ }^{* * *} P<0.001$ versus H9-NC. ${ }^{\#} P<0.05$, \#\#\# $P<$ 0.001 versus H9-ENDO, H9-MESO, or H9-ECTO.

significantly downregulated after IFI16 expression (Fig. 4h and Supplementary Fig. 8g-i). In the meanwhile, SOX17, FOXA2, Brachyury, SNAI2, and PAX6 were significantly upregulated (Fig. $4 \mathrm{~h}$ and Supplementary Fig. $8 \mathrm{~g}-\mathrm{i}$ ). Taken together, the results above suggest that IFI16 overexpression accelerates the downregulation of self-renewal gene expression and upregulation of the differentiation gene expression in the early stage of trilineage induction. 
IFI16 interacts with p53 during trilineage specification

The results above suggest the important role of IFI16 in the regulation of hESCs self-renewal and differentiation. To investigate the possible mechanism underlying this regulation, we assessed p53 expression since p53 has been considered as the downstream signal of IFI16. As shown in Fig. 5a-f, p53 had a relatively low expression level in $\mathrm{H} 9$ cells and was remarkably increased during trilineage specification, though the pattern of its upregulation was different in different germ layers. Notably, IFI16 knockdown did not significantly change the pattern of $\mathrm{p} 53$ expression during trilineage specification (Fig. 5a-f), indicating that the IFI16 role in the regulation of hESCs self-renewal and differentiation was not attributed to p53 expression. Since p53 could interact with IFI16 and mediated IFI16 function ${ }^{20}$, we were thereafter encouraged to verify the IFI16-p53 interaction during trilineage specification and investigate whether this interaction contributed to hESCs selfrenewal and differentiation. To ensure relatively high expression levels of p53 and IFI16, we performed co-immunoprecipitation (coIP) on Day 2 of trilineage induction. Indeed, solid interaction between p53 and IFI16 was observed during trilineage specification (Fig. $5 \mathrm{~g}, \mathrm{~h}$ ). On the contrary, rare IFI16-p53 interaction was found in $\mathrm{H} 9$ cells due to its relatively low levels of p53 and IFI16 (Fig. 5g, h). Consistently, results from proximity ligation assay (PLA) also demonstrated that IFI16 could interact with p53 in the nuclei of trilineage cells in situ, while the fluorescence signals were not found in $\mathrm{H} 9$ cells (Fig. 5i). Together, these results provide the evidence that IFI16 interacts with p53 during trilineage specification.

\section{IFI16 facilitates p53 binding in the genome}

To determine the roles of IFI16-p53 interaction during trilineage specification, we performed ChIP-seq to derive a genome-wide profile of p53-binding genes after IFI16 knockdown by sh1865. p53 binding sites were mapped on Day 2 of trilineage induction, when IFI16-p53 interaction was observed. After peak-calling, 11563,15697 , and 8832 p53 peaks were identified in endoderm, mesoderm, and ectoderm lineage induction of shNC group, while 3255,2158 , and 1795 p53 peaks were obtained, respectively, in sh1865 group (Fig. 6a). And the 10247, 14835, and 7139 special peaks in each lineage of shNC group were considered as IFI16 regulated p53 peaks (Fig. 6a). These results provided the initial evidence that IFI16 knockdown reduces p53-binding in the genome during trilineage specification. To validate whether the identified peaks contain p53 response elements, de novo motif analysis was conducted using MEME. We compared the sequences from the highly enriched peaks with the p53 binding motif sequences in the JASPAR database and found these sequences were enriched with p53 binding motifs (Supplementary Fig. 9a).

In addition, consistent with the findings from previous studies, the majority of p53-binding sites in the differentiated trilineage genome were localized in introns ${ }^{34}$. Other p53-binding sites are mainly scattered in the intergenic regions ( $>2 \mathrm{~kb}$ upstream/ downstream of the transcription start sites), within $2 \mathrm{~kb}$ upstream of transcriptional start site (TSS), within $2 \mathrm{~kb}$ downstream of transcriptional end site (TES) and exons. No evident differences were observed in the distribution of p53-binding sites among different lineage differentiation (Fig. 6b). However, IFI16 knockdown remarkably reduced the distribution ratio of p53 peaks within $2 \mathrm{~kb}$ upstream of TSS (Fig. 6b), which indicated that IFI16 mainly stabilizes the $\mathrm{p} 53$-binding pattern in the promoter regions during the different lineage differentiation of hESCs.

To investigate the biological significance of the IFI16 regulated p53 peaks during trilineage specification, we referred these p53 peaks to specific RefSeq genes if the peaks were within $2 \mathrm{~kb}$ of the genes, and identified 4806,6245 , and 4186 genes with p53 peaks in the endoderm, mesoderm, and ectoderm lineage, respectively (Fig. 6c, d). Consistently, the numbers of the p53-binding genes dropped to 1695,1107 , and 940 after IFI16 knockdown (Fig. 6c, d), and we defined the 3834,5442 , and 3277 genes associated special peaks in shNC groups as IFI16-p53 regulated genes. By using Cytoscape analysis for biological processes gene ontology (GO) terms, the biological functions of these p53-binding genes were further annotated. As expected, the genes involved in biological processes associated with p53 function, for example, "cell death" and "cell cycle" were enriched in the differentiated trilineages of shNC (Supplementary Fig. 9b-d). In addition, we found significant enrichment for genes contributes to development including "anatomical structure development" and "developmental process" (Supplementary Fig. 9b-d). Notably, "signal" and "regulation of gene expression" related genes were also observed in IFI16-p53 regulated gene group of differentiated trilineages (Fig. 6e-g). Moreover, "digestive tract development" and tepithelium development" was specially enriched for endoderm, "immune system development" and ecirculatory system development" for mesoderm and "nervous system development" and us systedifferentiation" for ectoderm (Fig. 6e-g). Together, the results above point to a possibility that IFI16 stabilizes p53-binding in the genome which probably contributes to trilineage specification of hESCs.

IFI16-p53 interaction differentially regulates of self-renewal and differentiation gene expression

We then explored whether the IFI16 regulated p53 peaks would contribute to the transcription regulation of the nearby genes. We performed RNA-seq and identified 3877, 4209, and 603 differential genes, respectively, in the endoderm, mesoderm, and ectoderm lineage after IFI16 knockdown (Fig. 7a). Moreover, significant enrichment for genes contributes to development including "anatomical structure development", "regulation of developmental process" and "animal organ development" were observed in these differential genes using Cytoscape analysis (Supplementary Fig. $10 a-c)$, which is consistent with the finding above that IFI16 knockdown inhibits trilineage specification. After integrating the IFI16 regulated p53 peaks with differential gene expression results, 656,1038 , and 92 genes were determined to be p53 targeting genes (Fig. 7a), which have one p53-binding peak at least and RNA level changed in the endoderm, mesoderm, and ectoderm lineage after IFI16 knockdown.

Notably, besides correlating with the upregulation of genes (termed p53 activated genes), binding of p53 probably also represses gene expression (termed p53 repressed genes) which include some key transcription regulators in hESCs (Fig. 7b and Supplementary Fig. 10d). The expression of FGF8 was reduced associated with decreased p53 peaks during trilineage specification (Fig. 7b). Moreover, the expression of endoderm marker genes, for example, GATA4, GATA6, and HNF4A were specifically downregulated in the endoderm lineage after IFI16 knockdown (Fig. 7b). Meanwhile, the expression of mesoderm marker gene LEF1 and ectoderm marker gene PCLO, OTX2 also decreased in the mesoderm and ectoderm lineage, respectively (Fig. 7b), correlated with the reduced p53 peaks. Especially, the expression levels of OCT4, NANOG, KLF4, and ZFP42 were upregulated when p53 peaks decreased (Fig. 7b), indicating that IFI16-p53 would inhibit self-renewal gene expression. In addition, genes related to Wnt signaling, MAPK signaling, apoptosis and p53 signaling were also regulated by IFI16-p53 interaction (Fig. 7b).

To further investigate the possible mechanism underlying the differential gene transcriptional outcome, i.e., activation or repression regulated by $\mathrm{p} 53$, we compared the p53 binding patterns within the p53 activated and repressed genes. As shown in Fig. 7c, we evaluated the $\mathrm{p} 53$ peaks in gene body, distal (2-100 kb upstream of the TSS), promoter-proximal (within $2 \mathrm{~kb}$ upstream of TSS) and proximal downstream (within $2 \mathrm{~kb}$ downstream of TES) regions and did not found an obvious difference in p53 peaks of gene body regions between p53 activated and repressed genes. Meanwhile, the differential binding of the p53 in the promoter and proximal 

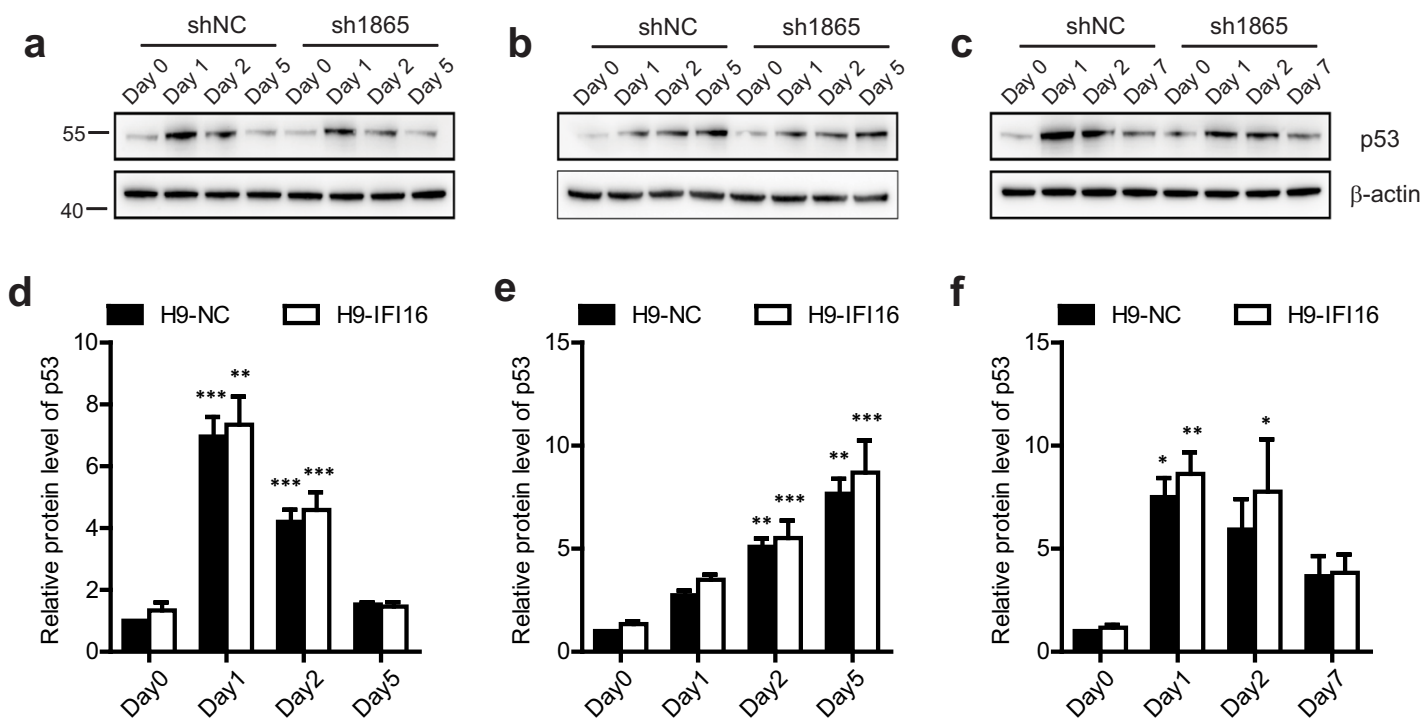

$\mathbf{f}$

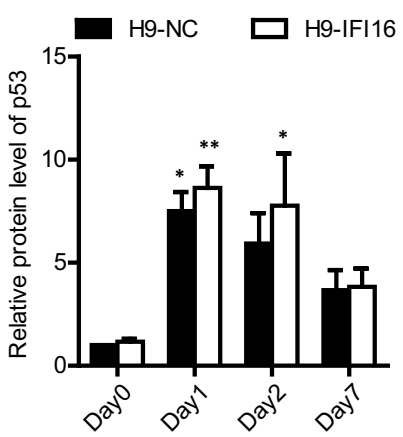

g

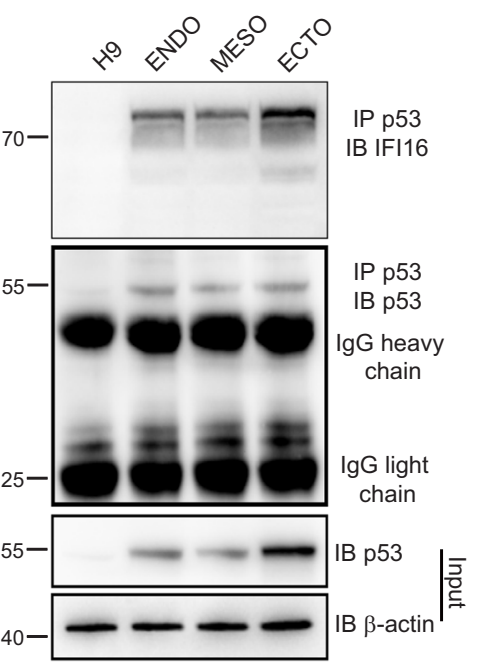

i

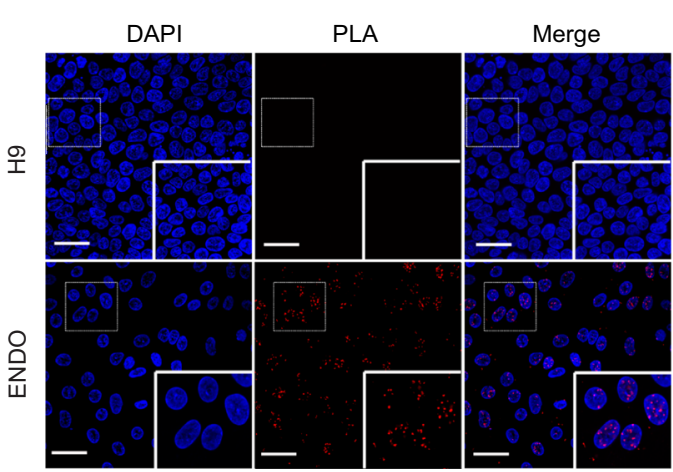

h

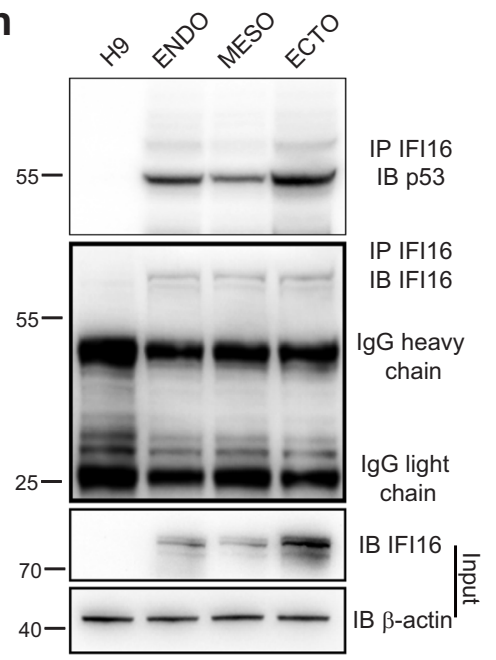

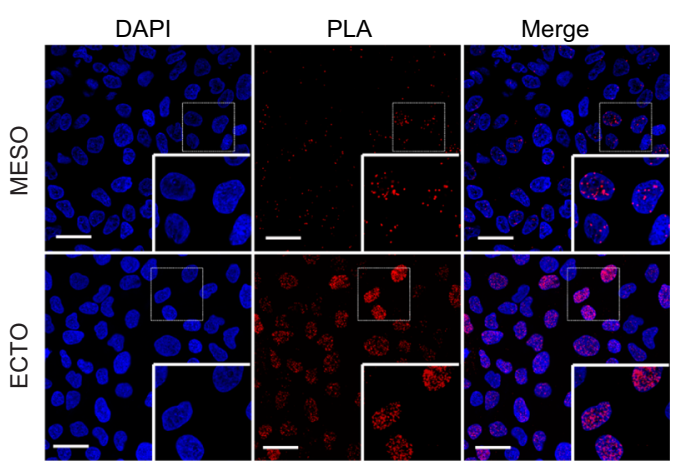

Fig. 5 IFI16 interacts with p53 during trilineage specification. a-c Representative immunoblots of p53 from H9 cells and differentiated trilineage (a, endoderm; b, mesoderm; c, ectoderm) infected with sh1865 or shNC. $\mathbf{d}$-f Statistics for $\mathbf{a}-\mathbf{c}$ respectively, $n=4$ for each group. The relative protein level of p53 was calculated relatively to which in H9-shNC group. $\mathbf{g}-\mathbf{h}$ lysates of $\mathrm{H} 9$ cells and differentiated trilineage on Day 2 precipitated with p53 (g) and IFI16 (h) antibodies, and immunoblotted with indicated antibodies. $\beta$-actin serves as a loading control. i PLA analysis of IFI16-p53 interaction in $\mathrm{H} 9$ cells and differentiated trilineage on Day 2. DAPI serves as a nucleus indicator. Dashed boxes indicate the zoom in reagions in each image. Scale bar, $50 \mu \mathrm{M}$. ENDO, endoderm; MESO, mesoderm; ECTO, ectoderm. NC, negative control. All data were presented as mean \pm SEM. Comparisons between groups for statistical significance were performed with two-way ANOVA with Bonferroni post hoc test $(\mathbf{d}-\mathbf{f}) .{ }^{*} P<0.05,{ }^{* *} P<0.01,{ }^{* *} P<0.001$ versus H9-NC Day 0.

downstream regions was not consistent among the trilineages (Fig. 7c, d). However, more p53 peaks were found in the distal regions of p53 activated genes (Fig. 7c, d), which indicating that the binding of p53 in the distal upstream region would contribute to the activation of gene expression. Since enhancers are usually located in the distal upstream region of genes and facilitated the transcription activation, the alignment of $\mathrm{p} 53$ peaks in distal upstream region (2 to $100 \mathrm{~kb})$ of p53-activated and p53-repressed genes with the validated enhancer 
a

shNC special peaks shNC-1865 shared peaks sh1865 special peaks

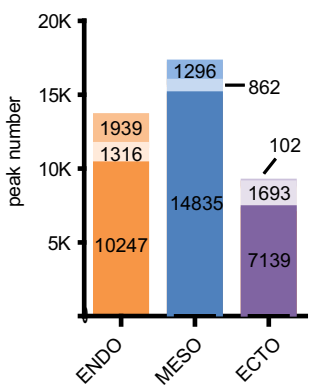

b

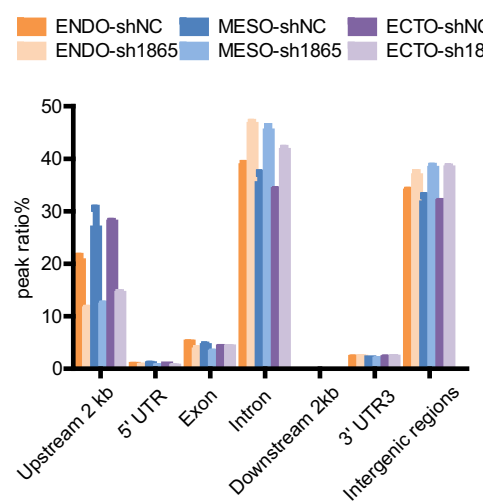

\section{e}

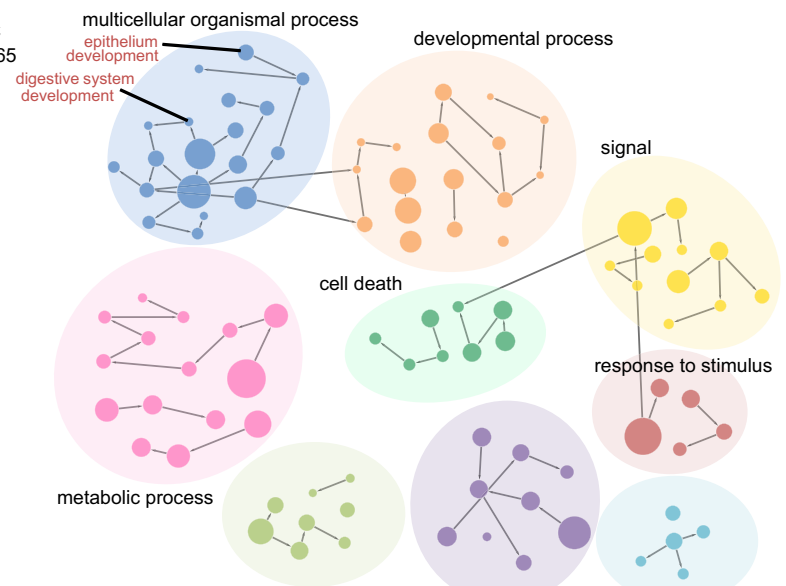

cell cycle

C
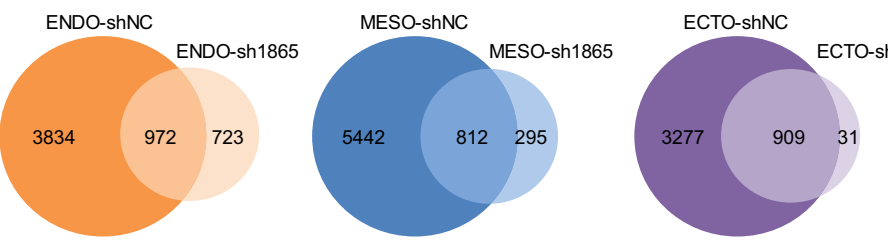

d

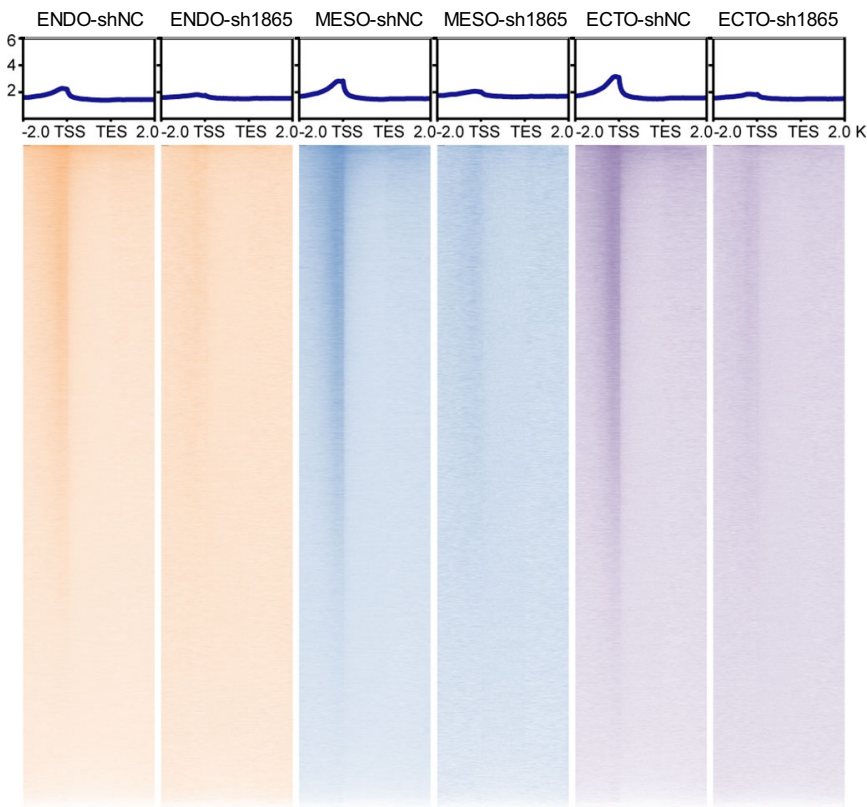

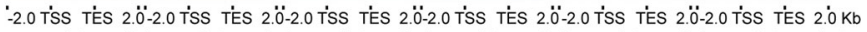
gene distance $(b p)$ gene distance (bp) gene distance (bp) gene distance (bp) gene distance (bp) gene distance (bp)

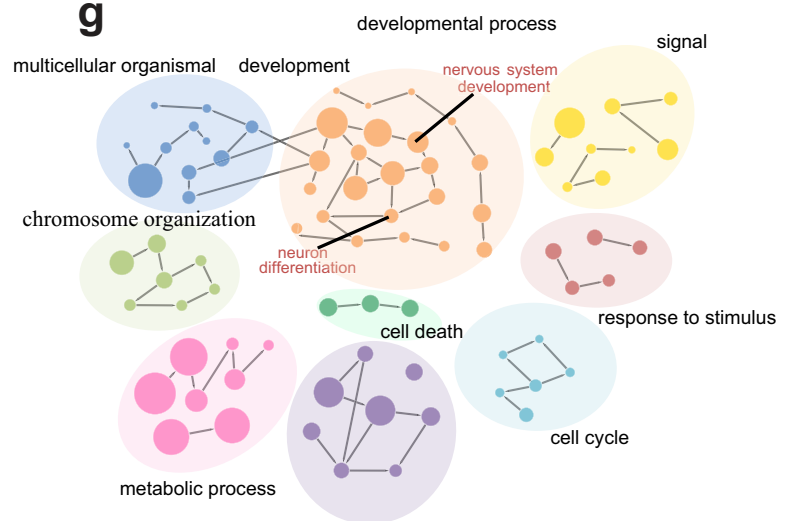

regulation of gene expression

Fig. 6 Genomic profiling of p53 peaks after IFI16 knockdown during trilineage specification. a Histogram of p53 peak number after IFI16 knockdown during trilineage specification. b The distribution ratio of p53 peaks after IFI16 knockdown during trilineage specification. c, d Venn diagrams (c) and heatmaps (d) of genes enriched by p53 peaks after IFI16 knockdown during trilineage specification. e-g Biological processes gene ontology (GO) analysis for genes enriched by p53-shNC special peaks in differentiated trilineage (e, endoderm; $\mathbf{f}$, mesoderm; g, ectoderm). Each node represented a specific GO term, and the node size indicated the number of genes in the GO term. The GO term annotations are listed in the Supplementary Dataset 1. ENDO, endoderm; MESO, mesoderm; ECTO, ectoderm.

sequences in the FANTOM5 were performed to identify the potential p53 binding enhancers. As shown in Fig. 7e, p53 peaks in the distal upstream region of $\mathrm{p} 53$-activated genes greatly enriched more overlapped enhancers, compared with p53-repressed genes, which suggested that p53 binding enhancers in the distal upstream region determine the transcription fate of $p 53$ binding genes. Collectively, all these results are consistent with an explanation that the upregulated expression of IFI16, which interacts with p53, would facilitate p53-binding in the genome to contribute to differentially regulation of self-renewal and differentiation gene expression and further determine the trilineage specification of hESCs (Fig. 7f). 

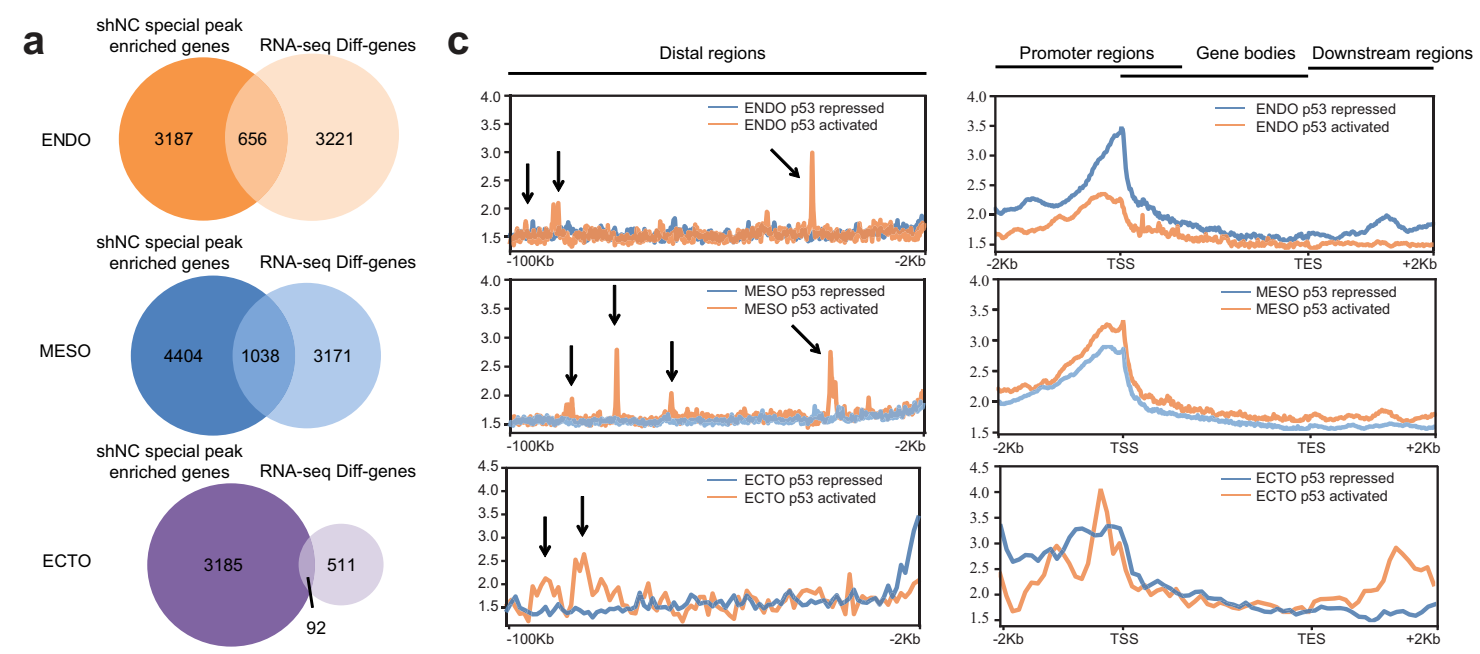

b

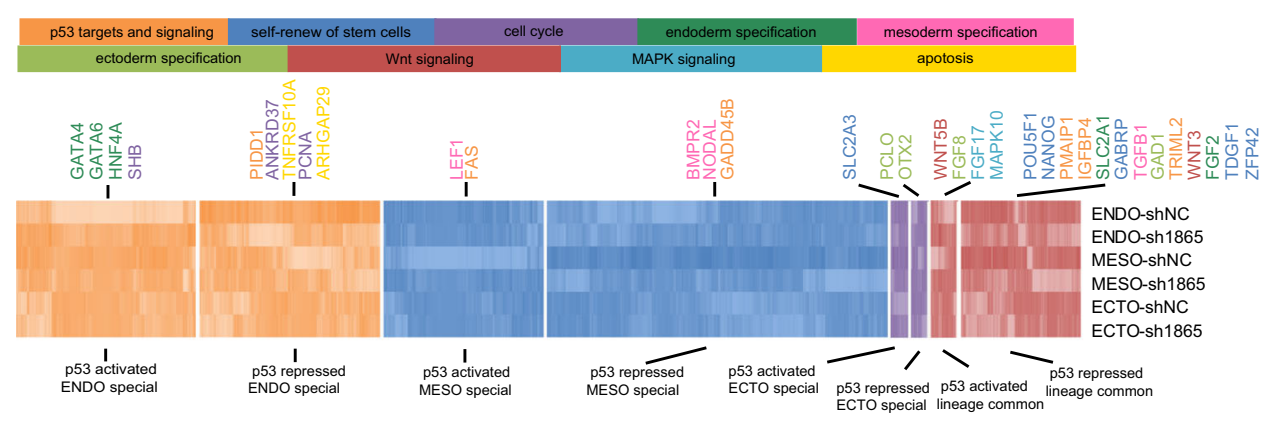

d
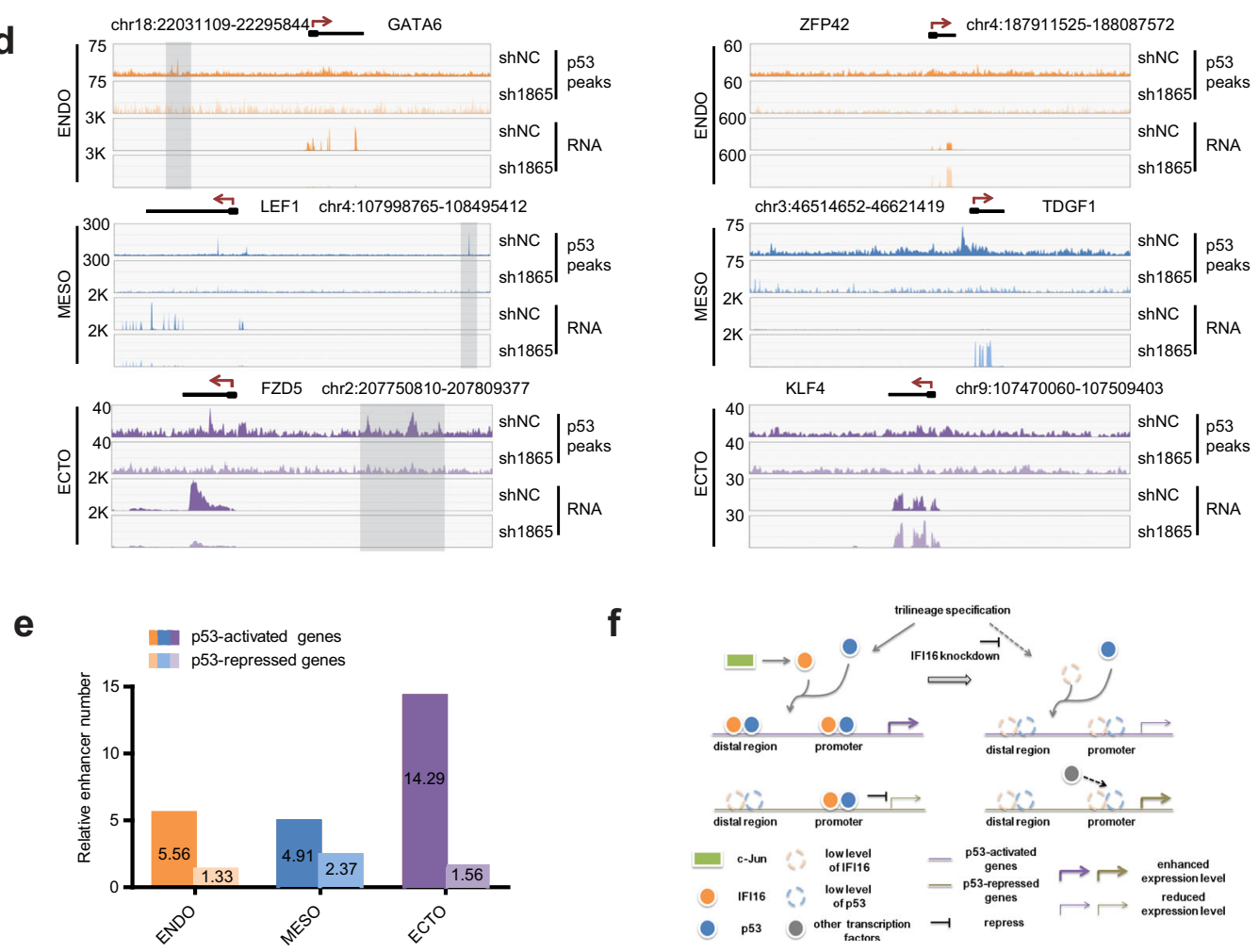

\section{DISCUSSION}

Here, we report a novel mechanism by which IFI16-p53 interaction promotes hESCs differentiating into trilineage cells. Several lines of evidence support this conclusion. Firstly, IFI16 expression levels were upregulated through JNK activation during trilineage

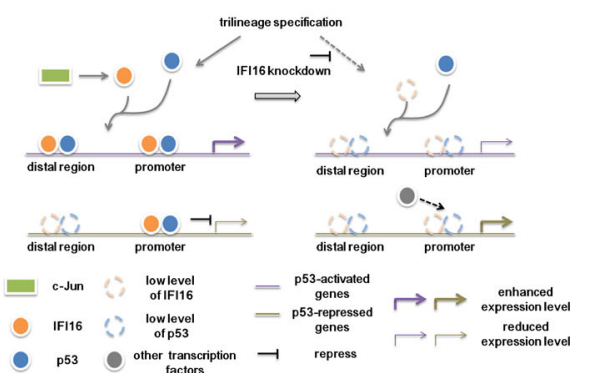

specification. Secondly, IFI16 knockdown delays the downregulation of self-renewal gene expression, such as OCT4, SOX2, and KLF4 and suppresses the upregulation of differentiation gene expression including SOX17, FOXA2, CXCR4, Brachyury, PAX6, and OTX2. Thirdly, IFI16 overexpression accelerates trilineage 
Fig. 7 Differentially regulating self-renewal and differentiation gene expression by p53-shNC special peaks. a Venn diagrams of $\mathrm{p} 53$-shNC special peak enriched genes and genes differentially expressed after IFI16 knockdown during trilineage specification. b Heatmaps (bottom) of genes specially and commonly regulated by p53-shNC special peaks after IFI16 knockdown and corresponding function/pathway (up) during trilineage specification. c Averaged p53 peaks in distal, promoter and gene body, and proximal downstream regions of p53-activated and p53repressed genes in differentiated trilineage. The arrows indicate the averaged p53 peaks in distal regions of p53-activated genes. d Representative genomic views of p53 peaks and RNA levels at p53-activated (left) and p53-repressed (right) genes after IFI16 knockdown in differentiated trilineage. The shadow boxes indicate significant p53 peaks in the shNC group. e Histogram of the relative numbers of enhancer overlapped with p53 peaks in the TSS upstream ( 2 to $100 \mathrm{~kb})$ of p53-activated and p53-repressed genes in differentiated trilineage. f Schematic diagram depicting a working model for IFI16-p53 interaction differentially regulating gene expression during trilineage specification. ENDO, endoderm; MESO, mesoderm; ECTO, ectoderm.

specification through downregulation of self-renewal gene expression and upregulation the of differentiation gene expression. Lastly, IFI16 stabilizes p53-binding in the genome which correlated with the self-renewal and differentiation gene expression during hESCs trilineage specification. Together, our results suggest a particular role of IFI16 in differential gene expression regulation which is dependent on the genome-wide profile of p53-binding facilitated by IFI16-p53 interaction. Therefore, IFI16 could be a potential target to interfere in both the self-renewal and differentiation regulatory network of hESCs.

It has been mentioned above that the transcriptional regulatory network of hESCs was well studied. However, how differentiation signals disrupt the self-renewal network and further determine trilineage fate of hESCs remains largely unknown. An important implication of the current findings is that a key transcriptional factor globally regulates both self-renewal and differentiation genes contributing to the trilineage fate determination of hESCs. Here, JNK activation induces the upregulation of IFI16 expression, which leads to the enhancement of IFI16-p53 interaction. Furthermore, this interaction is suggested to activate some differentiation genes including GATA4, GATA6, LEF1, BMPR2, PCLO, and GAD1 and inhibit some self-renewal genes, such as OCT4, NANOG, KLF4, and ZFP42. Therefore, it indicates that transcriptional factors with global binding sites like p53 would regulate the expression of numerous genes and probably play crucial roles in both the self-renewal and differentiation regulatory network of hESCs.

The large number of transcriptional regulatory regions throughout the human genome dynamically interacts to control the expression of millions of base pairs ${ }^{35,36}$. Particularly, the expression of specific genes in different cell types is usually regulated by enhancer and promoter interaction ${ }^{37,38}$. Transcription factors and RNA polymerase II bind to both enhancer and promoter to mediate the interactions and control the gene transcription ${ }^{38}$. Here, one of the important findings of this study is that the p53binding in some validated enhancers correlated with the activation of gene expression. Since p53 functions as a tetramer ${ }^{39}$, it points to a possibility that $\mathrm{p} 53$ binds to both enhancer and promoter of the genes and sequentially mediates enhancerpromoter loops to drive the transcriptional activation. Therefore, examination of the role of p53 in enhancer-promoter interaction formation using chromatin interaction analysis using paired-end tag sequencing (ChIA-PET) ${ }^{40}$ or in situ $\mathrm{Hi}-\mathrm{C}$ followed by chromatin immunoprecipitation (HiChIP) ${ }^{41}$ would further expand our understanding of the mechanism underlying the differential regulation gene expression by IFI16-p53 interaction during hESCs trilineage specification.

IFI16 has important roles in diverse biological processes, including intracellular DNA sensing, antiviral restriction, and cell cycle regulation, while little is known about its role of differentiation regulation in hESCs. As the DNA binding of IFI16 was not dependent on the sequence specificity, specific gene regulation by IFI16 largely was relied on its interaction with other proteins containing specific DNA binding motifs ${ }^{7,42,43}$. A novel finding of our study is that IFI16 interacts with p53 and stabilizes p53binding in the genome to differentially control gene expression.
Notably, IFI16 expression levels were continually potentiated during hESCs trilineage specification. In the meanwhile, p53 expression levels were differentially regulated in the different lineage. Therefore, it is likely that other proteins in trilineage, especially in endoderm and ectoderm, also interacts with IFI16 and play its role in cell differentiation. Indeed, IFI16 also has a relatively high expression level in the skin, urinary bladder, and nasopharynx (https://www.proteinatlas.org/). Identifying other IFI16-interacting protein would provide us new insights into the physiological roles of IFI16 in embryogenesis or even somatic cells.

Collectively, our results point to a previously unknown role of IFI16-p53 interaction in regulating both self-renewal and differentiation gene expression and uncover the genome-wide profiling of p53 targets and corresponding gene expression during hESCs differentiation, which expands our understanding of the fate determination of hESCs.

\section{METHODS}

hESCs culture and trilineage specification

Human embryonic stem cell (hESC) line H9 (order number: 18-1-1522) was from Cell Bank of the Shanghai Institutes for Biological Sciences of the Chinese Academy of Sciences and authenticated using Short Tandem Repeat (STR) analysis (GENETIC TESTING BIOTECHNOLOGY Co., Ltd.). hESCs were maintained in feeder-free cell culture medium mTeSR ${ }^{\mathrm{T}}$ 1 (STEMCELL Technologies, \#85850). hESCs were passaged every 5-6 days using ReLeSR $^{\text {TM }}$ (STEMCELL Technologies, \#05873) and had maintained a stable karyotype even until the 50th generation (Beijing Cellapybio Biotechnology Co., Ltd.).

Trilineage endoderm, mesoderm, and ectoderm differentiation was performed using STEMdiff ${ }^{\mathrm{TM}}$ Trilineage specification Kit (STEMCELL Technologies, \#05230). Briefly, hESCs were dissociated into single cells by TrypLE $^{\mathrm{TM}}$ (ThermoFisher, 12604021) and resuspended in DMEM/F-12 (ThermoFisher, 11330057). After centrifuging at $300 \times \mathrm{g}$ for $5 \mathrm{~min}$, cell pellets were resuspended in $\mathrm{mTeSR}^{\mathrm{TM}} 1 /$ Ectoderm Medium with $10 \mu \mathrm{M} \mathrm{Y}$ 27632. The Endoderm /Mesoderm/ Ectoderm Medium was replaced once a day. Endoderm and mesoderm lineages were harvested at day 5, while ectoderm lineage was harvested at day 7.

\section{Lentivirus package and infection}

To package the sh1865 and sh2153 lentiviruses, the IFI16 target sequence (listed in Supplementary Table 1) was inserted into the hU6-MCS-CMVPuromycin (GV112) plasmid. After transfecting HEK293T cells with sh1865/ sh2153 GV112 with helper plasmids, the supernatant was collected at $48-72 \mathrm{~h}$. Sequentially, the supernatant was filtered and centrifuged to harvest virus particles. The sh1865 and sh2153 lentiviruses were introduced to hESCs at MOI of 100. After infection, the sh1865 and sh2153 hESC lines were obtained by using $1 \mu \mathrm{g} / \mathrm{ml}$ puromycin.

To package the IFI16 overexpression lentivirus, the IFI16 sequence (NM_001206567, listed in Supplementary Table 1) was inserted into the TetIIP-MCS-3FLAG-Ubi-TetR-IRES-Puromycin (GV308) plasmid. After transfecting HEK293T cells with sh1865/sh2153 IFI16 overexpression GV308 with helper plasmids, the supernatant was collected at $48-72 \mathrm{~h}$. Sequentially, the supernatant was filtered and centrifuged to harvest virus particles. The IFI16 overexpression lentivirus was introduced to hESCs at $\mathrm{MOI}$ of 300 . After infection, the IFI16 overexpression hESC lines were obtained by using $1 \mu \mathrm{g} / \mathrm{ml}$ puromycin. And the IFI16 expression was induced by $1 \mu \mathrm{g} / \mathrm{ml}$ doxycycline. 


\section{Immunofluorescence and image analysis}

The prepared cells were washed twice with $0.1 \mathrm{mM}$ phosphate-buffered saline (PBS) and then crosslinked by $4 \%$ paraformaldehyde for 20 mins at room temperature. After another wash with $0.1 \mathrm{mM}$ PBS, the cells were incubated with $10 \%$ BSA and $0.5 \%$ Triton X-100 in PBS for $1 \mathrm{~h}$. Primary antibodies (anti-IFI16 1:500, anti-AIM2 1:1000, anti-OCT4 1:1000, anti-SOX2 1:1000, anti-SOX17 1:1000, anti-FOXA2 1:500, anti-Brachyury 1:1000, antiSNAI2 1:400, and anti-PAX6 1:1000) or isotypes (mouse lgG1/rabbit lgG 1:1000) were then added and incubated at $4^{\circ} \mathrm{C}$ overnight. The next day, the cells were washed with $0.1 \mathrm{mM}$ PBS three times and followed by incubation with secondary antibodies $(1: 1000)$ conjugated with a fluorophore at room temperature for $2-3 \mathrm{~h}$ The nucleus was then stained by using 4,6-Diamidino-2-phenylindole (DAPI). The fluorescence expression of OCT4, SOX2, SOX17, FOXA2, Brachyury, SNAI2, and PAX6 was defined as the average optical density (AOD) of immunoreactivity, which was quantified by ImageJ. Briefly, the integrated optical intensity of target gene was measured and then the background immunoreactivity was subtracted prior to analysis. For each sample, the background immunoreactivity was defined as the integrated optical intensity of the isotype (mouse lgG1/rabbit lgG) staining (Supplementary Figs. 11-13). The AOD in each sample was obtained by calculating the value of the average optical density normalized by integrated optical intensity of DAPI in the same field as following:

$\mathrm{AOD}=\mathrm{IDT} / \mathrm{IDDT}-\mathrm{IDI} / \mathrm{IDDI}$

Where IDT is the integrated intensity of target gene, IDI is the integrated intensity of IgG and IDD is the integrated intensity of DAPI for target gene (IDDT) and isotype (IDDI).

\section{Flow cytometry}

The marker protein of trilineage analyzed by flow cytometry was conducted as the manufacturer's instructions of Fixation/Permeabilization Solution Kit (BD Biosciences, 554714). Briefly, the cultured cells were dissociated into single cells and resuspended in $0.1 \mathrm{mM}$ PBS. After centrifuging at $300 \times g$ for $5 \mathrm{~min}$, cells were incubated with fixation/ permeabilization solution for $20 \mathrm{~min}$ at room temperature. Sequentially, the diluted BD Perm $/$ Wash $^{\text {TM }}$ Buffer was used to wash the cells twice. Then antibodies of marker protein (endoderm: SOX17-APC, FOXA2-488; mesoderm: Brachyury-APC, CXCR4-FITC; ectoderm: Nestin-APC, PAX6-488) or isotypes (mouse IgG2A-FITC, goat IgG-APC, goat lgG-488, mouse IgG-APC, mouse IgG2a kappa-488, Supplementary Fig. 14) were then added for staining. After staining at room temperature for $30 \mathrm{~min}$, flow cytometry was then performed to analyze the $488 / \mathrm{FITC}^{+}$and/or $\mathrm{APC}^{+}$population. To evaluate the role of IFI16 in trilineage specification, the proportion of SOX $17^{+} / \mathrm{FOXA2}^{+}$, Brachyury ${ }^{+} / \mathrm{CXCR}^{+}{ }$, Nestin ${ }^{+} / \mathrm{PAX}^{+}$cells was statistically analyzed after IFI16 knockdown and overexpression.

\section{RNA isolation, reverse transcription, and $\mathrm{qPCR}$}

Total RNA was extracted from the harvested cells by using Trizol (ThermoFisher, 15596026) and the concentration of the total RNA was measured by NanoDrop 2000 (ThermoFisher). The CDNA was then reversetranscribed by using PrimeScript RT reagent Kit with gDNA Eraser (Takara, RR047A) from $1 \mu \mathrm{g}$ RNA. The expression levels of genes were quantified by quantitative real-time PCR (qPCR) using AceQ Universal SYBR qPCR Master Mix (Vazyme, Q511-02). The reaction condition of qPCR: Denaturation at $95^{\circ} \mathrm{C}$ for 2 mins. Denaturation at $94^{\circ} \mathrm{C}$ for $15 \mathrm{~s}$, annealing and extension at $60^{\circ} \mathrm{C}$ for $30 \mathrm{~s}, 40$ cycles. Quantification was analyzed by using the comparative $\mathrm{Ct}(\Delta \Delta \mathrm{Ct})$ method. The expression levels of genes were normalized by the housekeeping gene (GAPDH). The primer sequences for qPCR were listed in Supplementary Table 1.

\section{Western blots}

The harvested cells were lysed in a lysis buffer (50 mM Tris- $\mathrm{HCl}(\mathrm{pH} 6.8), 2 \%$ sodium dodecyl sulfate, 1.5\% DL-Dithiothreitol, $10 \%$ glycerol and $0.2 \%$ Bromophenol blue), and denatured at $100^{\circ} \mathrm{C}$ for 10 mins. Sequentially the total extracts were subjected to SDS-PAGE using 4-20\% gradient gel (GenScript, M00655) and further transferred to Immobilon-FL PVDF (Millipore, IPFL85R). After blocking with Protein Free Rapid Block Buffer (EpiZyme, PS108), the protein was incubated with the primary antibodies (anti-IFI16 1:500, anti-AIM2 1:1000, anti-p53 1:500, anti-OCT4 1:1000, antiSOX2 1:1000, anti-SOX17 1:1000, anti-FOXA2 1:1000, anti-Brachyury 1:1000, anti-OTX2 1:1000, anti-PAX6 1:1000, and anti- $\beta$-actin $1: 5000$ ) at $4{ }^{\circ} \mathrm{C}$ overnight. The next day, the protein was washed with Tris-buffered saline $+0.1 \%$ Tween-20 (TBST) three times and then incubated with secondary antibodies for $2 \mathrm{~h}$ at room temperature. The expression of protein was detected by chemiluminescence imaging system (GeneGnomeXRQ, SYNGENE) and analyzed by densitometry using Image J. The background correction was done with the value of 50 (called rolling disc in the software). To calculate the relative expression of specific protein, the $\beta$-actin serves as a reference for the sample loading. All blots derive from the same experiment and were processed in parallel. The uncropped blots are listed in the Supplementary Figs. 15-23.

\section{Co-Immunoprecipitation}

The hESCs and differentiated trilineage cells were cultured in a $10 \mathrm{~cm}$ dish for 2 days. Sequentially, the cells were harvested and lysed in Pierce ${ }^{\mathrm{TM}} \mathrm{IP}$ Lysis Buffer (ThermoFisher, 87787). After rotation at $4^{\circ} \mathrm{C}$ for $1 \mathrm{~h}$, the cell lysates were incubated with p53 or IFI 16 antibodies at $4{ }^{\circ} \mathrm{C}$ overnight. The next day, the protein A/G magnetic beads (Biotool, b23202) were added to capture the protein-antibody complex at $4^{\circ} \mathrm{C}$ for $3 \mathrm{~h}$ After washing twice with Pierce ${ }^{\mathrm{TM}}$ IP Lysis Buffer and a final wash with TBST, the magnetic beads were incubated in western blots lysis buffer at $100^{\circ} \mathrm{C}$ for 10 mins to elute the target protein. Lastly, the eluted products were subjected to western blots analysis.

\section{PLA technology}

The in situ p53-IFI16 interaction was detected using Duolink ${ }^{\circledR}$ In Situ Red Starter Kit Mouse/Rabbit (Sigma, DUO92101) according to the manufacturer's instructions. Briefly, the cultured cells were washed with $0.1 \mathrm{mM}$ PBS and then crosslinked by $4 \%$ paraformaldehyde for $15 \mathrm{~min}$ at room temperature. After blocking the cells with the Duolink ${ }^{\otimes}$ Blocking Solution for $1 \mathrm{~h}$ at $37^{\circ} \mathrm{C}$, primary antibodies (anti-IFI16 1:500 and anti-p53 1:500) were then added and incubated at $4{ }^{\circ} \mathrm{C}$ overnight. The next day, the cells were washed with $2 \times 5$ mins in $1 \times$ Wash Buffer $A$ at room temperature and followed by incubation with the proximity ligation assay (PLA) probe solution at $37^{\circ} \mathrm{C}$ for $1 \mathrm{~h}$. After another $2 \times 5$ min-wash with $1 \times$ Wash Buffer $\mathrm{A}$, the ligation solution was applied at $37^{\circ} \mathrm{C}$ for $30 \mathrm{~min}$. Sequentially, the ligation solution was removed and the cells were then washed with $2 \times$ 5 mins in $1 \times$ Wash Buffer A. After incubation with the amplification at $37^{\circ} \mathrm{C}$ for $2 \mathrm{~h}$, the final wash with $1 \times$ Wash Buffer B was performed. The nucleus was then stained by using Duolink ${ }^{\circledR}$ In Situ Mounting Medium with DAPI. The fluorescence signals were detected using a confocal microscope (Leica, TCS SP8 X).

\section{Alkaline phosphatase staining}

The alkaline phosphatase staining was conducted as the protocol of Alkaline Phosphatase Kit (Sigma, 85L2). Briefly, the cultured cells were fixed using the Fixative Solution for $30 \mathrm{~s}$ at room temperature. After rinsing gently in deionized water for $45 \mathrm{~s}$, the alkaline-dye mixture was applied at room temperature for another $30 \mathrm{~min}$. Sequentially, the cells were rinsed gently in deionized water for $2 \mathrm{~min}$ and then incubated with Mayer's Hematoxylin Solution for 10 mins at room temperature.

\section{CCK-8 assay}

The effect of IFI16 knockdown on cell viability was determined with Cell Counting Kit-8 (CCK-8) assay (MedChemExpress, HY-K0301). Briefly, the sh1865 and shNC hESC lines were dissociated into single cells and further induced into trilineage using STEMdiff ${ }^{\mathrm{M}}$ Trilineage specification Kit in 24well plates. The CCK-8 solution was added to each well of the plate at Day 0 , Day 1, Day 2, and Day 5 for the endoderm and mesoderm, Day 0, Day 1, Day 2, and Day 7 for the ectoderm. After incubation for $2 \mathrm{~h}$, the supernatant was collected and the absorbance was measured at $450 \mathrm{~nm}$.

\section{ChIP-seq}

The cultured cells (about 5 million) were crosslinked with $1 \%$ formaldehyde for $5 \mathrm{~min}$ at room temperature, and then quenched with $125 \mathrm{mM}$ glycine. After a wash with $0.1 \mathrm{mM}$ PBS, the cells were harvested and lysed in ChIP Lysis Buffer (0.1\% SDS, 1\% Triton X-100, 2 mM EDTA, $150 \mathrm{mM} \mathrm{NaCl}, 50 \mathrm{mM}$ Tris- $\mathrm{HCl} \mathrm{pH} \mathrm{8.0)} \mathrm{at} 4{ }^{\circ} \mathrm{C}$ for $1 \mathrm{~h}$ to lyse the cell membrane. After centrifuging at $2500 \times \mathrm{g}$ for $5 \mathrm{mins}$, the nuclei were collected and the chromatin DNA was sheared into $300-600 \mathrm{bp}$ fragments by the sonicator (protocol: $25 \%$ amplitude; duration of $15 \mathrm{~min} ; 30 \mathrm{~s}$ ON and $30 \mathrm{~s}$ OFF; JY99-IIDN, SCIENTZ). Sequentially, the supernatant was harvested after centrifuging at $12,000 \times g$ 
for 10 mins. The antibodies ( $\mathrm{p} 53$ or c-Jun, $10 \mu \mathrm{g}$ ) were added for incubation at $4{ }^{\circ} \mathrm{C}$ overnight. Next day, the protein $\mathrm{A} / \mathrm{G}$ magnetic beads were added to capture the protein-antibody complex at $4^{\circ} \mathrm{C}$ for $3 \mathrm{~h}$. After careful washes with Low Salt Wash Buffer (0.1\% SDS, 1\% Triton X-100, 2 mM EDTA, $50 \mathrm{mM}$ HEPES pH 7.9, $150 \mathrm{mM} \mathrm{NaCl})$, High Salt Wash Buffer (0.1\% SDS, $1 \%$ Triton X100, 2 mM EDTA, $50 \mathrm{mM}$ HEPES pH 7.9, $500 \mathrm{mM} \mathrm{NaCl}$ ), LiCl Wash Buffer (250 mM LiCl, 0.5\% NP40, 0.5\% sodium deoxycholate, $1 \mathrm{mM}$ EDTA, $20 \mathrm{mM}$ Tris- $\mathrm{HCl} \mathrm{pH} 8.0$ ), and TE Buffer (10 mM Tris- $\mathrm{HCl}$ pH 8.0, $1 \mathrm{mM}$ EDTA), the was ChIP products were eluted with ChIP Elution Buffer (1\% SDS, $10 \mathrm{mM}$ EDTA, $50 \mathrm{mM}$ Tris- $\mathrm{HCl} \mathrm{pH} 8.0$ ) at $65^{\circ} \mathrm{C}$ for $20 \mathrm{~min}$. Crosslinking was reversed in $65^{\circ} \mathrm{C}$ overnight and the protein was digested by proteinase $\mathrm{K}$ $(20 \mathrm{mg} / \mathrm{ml})$. ChIP-seq library construction and further sequencing was carried out by Annoroad Gene Technology. For ChIP-qPCR, the DNA binding levels of c-Jun at IFI16 promoter, AIM2 promoter, CGAS promoter and IFI16 $3^{\prime} \mathrm{UTR}$ during trilineage specification were calculated relatively to those of $\mathrm{H} 9$ cells. The c-Jun binding sites of AIM2 promoter and CGAS promoter is referred to the UCSC Genome Browser (http://genome.ucsc.edu/).

\section{RNA-seq}

For gene expression level measurement, total RNA was extracted from the hESCs and trilineage differentiated cells using TRIzol. RNA-seq library construction and further sequencing was carried out by Annoroad Gene Technology.

\section{ChIP-seq and RNA-seq data analysis}

After the quality and redundancy filtering, the sequenced tags were used for peak calling using the MACS2 algorithm with the settings for peak enrichment $>20$, peak-to-background enrichment $>3$, a kernel bandwidth of 300 . The p53 ChIP-seq peaks were analyzed using the MEME using a de novo motif analysis of the binding site sequences with default parameters. TOMTOM tool of MEME was used for alignment of the achieved DNA motifs with the identified motifs in the JASPAR database. The comparisons between the shNC and sh1865 p53 ChIP-seq peaks were performed using MAnorm with default parameters.

For the RNA-seq analysis, the alignments were performed using the STAR aligner, and the differential expression analysis was conducted using DESEQ2 as suggested to obtain a list of differentially regulated genes (Fold change $>1.5, \mathrm{FDR}<0.01$ ).

The biological functions of the genes derived from these p53 ChIP-seq peaks and differentially regulated genes during trilineage differentiation were annotated by using 'BiNGO' (Biological Network Gene Ontology) tool of Cytoscape for biological processes gene ontology (GO) terms, with significance threshold 0.01. Each node represented a specific GO term, and the node size indicated the number of genes in the GO term.

The enhancer identification was conducted using human enhancer database from FANTOM5 (http://fantom.gsc.riken.jp/papers/). Briefly, the p53 peaks of TSS upstream ( 2 to $100 \mathrm{~kb}$ ) of p53-activated and p53repressed genes in differentiated trilineage were harvested, respectively, to align with the validated enhancer sequences in the FANTOM5. The enhancer that overlaps with $\mathrm{p} 53$ peak was retained, and the enhancer that falls in the intron, exon and UTRs of genes was further rejected.

\section{Antibodies and reagents}

Antibodies were used as following: IFI16 was purchased from Abcam (ab169788, Western blots/Co-IP) and Santa Cruz (sc-8023, PLA/Immunofluorescence), p53 was purchased from CST (2527, PLA) and Santa Cruz (sc126, ChIP/Western blots/Co-IP), AIM2 was purchased from Abcam (ab180665, Western blots/Immunofluorescence), cGAS was purchased from CST (15102S, Western blots/ Immunofluorescence), OCT4 was purchased from Abcam (ab19857, Western blots/Immunofluorescence), SOX2 was purchased from CST (2748S, Western blots/Immunofluorescence), Brachyury was purchased from Abcam (ab20680, Western blots/ Immunofluorescence), OTX2 was purchased from Abcam (ab21990, Western blots/ Immunofluorescence), PAX6 was purchased from Abcam (ab5790, Western blots/ Immunofluorescence), SOX17 was purchased from Abcam (ab84990, Western blots/ Immunofluorescence), SNAI2 was purchased from Santa Cruz (sc-166476, Immunofluorescence), FOXA2 was purchased from R\&D (AF2400, Western blots/Immunofluorescence), c-Jun was purchased from Abcam (ab31419, Western blots), p38 was purchased from CST (9212s, Western blots), p-p38 was purchased from CST (9211s, Western blots), ERK was purchased from CST (4695s, Western blots), p-ERK was purchased from CST (4370s, Western blots), JNK was purchased from CST (9252s, Western blots), p-JNK was purchased from Santa Cruz (sc-6254, Western blots),
CXCR4-FITC was purchased from R\&D (FAB170F, Flow cytometry), BrachyuryAPC was purchased from R\&D (IC2085A, Flow cytometry), SOX17-APC was purchased from R\&D (IC1924A, Flow cytometry), FOXA2-488 was purchased from R\&D (IC2400G, Flow cytometry), Nestin-APC was purchased from R\&D (IC1259A, Flow cytometry), PAX6-488 was purchased from BD (561664, Flow cytometry), $\beta$-actin was purchased from ZEN BIO (200058-BF10, Western blots), rabbit IgG isotype control was purchased from CST (3900S, Immunofluorescence), mouse $\operatorname{lgG} 1$ isotype control was purchased from CST (5415S, Immunofluorescence), mouse IgG2A-FITC was purchased from R\&D (IC003F, Flow cytometry), goat IgG-APC was purchased from R\&D (IC108A, Flow cytometry), goat IgG-488 was purchased from R\&D (IC108G, Flow cytometry), mouse IgG1-APC was purchased from R\&D (IC002A, Flow cytometry), and mouse IgG2a kappa-488 was purchased from BD (565358, Flow cytometry).

Drugs were used as following: SP600125, SB203580, and U0126 were all purchased from Selleck Chemicals (S1460, S1076, and S1102).

\section{Statistical analysis}

Statistical analysis was carried out using GraphPad Prism 5.0 evaluate the differences between different groups. All data were presented as mean \pm SEM from three or more independent experiments. Comparisons between groups for statistical significance were performed with Student's $t$ test or analysis of variance (ANOVA) with post hoc test in multiple groups. Results were considered significant difference at ${ }^{*} P<0.05 ;{ }^{* *} P<0.01 ;{ }^{* * *} P<0.001$;

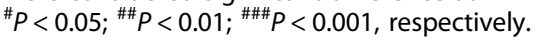

\section{Reporting summary}

Further information on research design is available in the Nature Research Reporting Summary linked to this article.

\section{DATA AVAILABILITY}

All relevant data of this study are available within the paper. ChIP-seq and RNA-seq raw data and processing data supporting this work have been deposited in Gene Expression Omnibus under the accession number GSE142050. Requests for further information should be directed to and will be fulfilled by Furong Li (frli62@163.com).

\section{CODE AVAILABILITY}

All studies used the commercial software in the generation or processing of datasets. Software and the corresponding version numbers were listed as following: ImageJ 1.46j, Photoshop CS6, Illustrator Adobe CS6, Graphpad Prism 5.0, FlowJo 10.4.2, MACS2 2.2.1, MEME 5.1.0, MAnorm 1.2.0, Cytoscape 3.7.1, DESEQ2 3.10, FANTOM5 5.

Received: 23 March 2020; Accepted: 25 September 2020; Published online: 29 October 2020

\section{REFERENCES}

1. Takahashi, K. \& Yamanaka, S. Induction of pluripotent stem cells from mouse embryonic and adult fibroblast cultures by defined factors. Cell 126, 663-676 (2006).

2. Boyer, L. A. et al. Core transcriptional regulatory circuitry in human embryonic stem cells. Cell 122, 947-956 (2005).

3. Pan, G. \& Thomson, J. A. Nanog and transcriptional networks in embryonic stem cell pluripotency. Cell Res. 17, 42-49 (2007).

4. Unterholzner, L. et al. IFI16 is an innate immune sensor for intracellular DNA. Nat. Immunol. 11, 997-1004 (2010).

5. Trapani, J. A. et al. A novel gene constitutively expressed in human lymphoid cells is inducible with interferon-gamma in myeloid cells. Immunogenetics 36, 369-376 (1992).

6. Trapani, J. A., Dawson, M., Apostolidis, V. A. \& Browne, K. A. Genomic organization of IFI16, an interferon-inducible gene whose expression is associated with human myeloid cell differentiation: correlation of predicted protein domains with exon organization. Immunogenetics 40, 415-424 (1994).

7. Iqbal, J. et al. Histone H2B-IFI16 recognition of nuclear herpesviral genome induces cytoplasmic interferon-beta responses. PLoS Pathog. 12, e1005967 (2016).

8. Roy, A., Ghosh, A., Kumar, B. \& Chandran, B. IFI16, a nuclear innate immune DNA sensor, mediates epigenetic silencing of herpesvirus genomes by its association with H3K9 methyltransferases SUV39H1 and GLP. Elife 8, https://doi.org/10.7554/ eLife.49500 (2019). 
9. Kim, E. J., Park, J. I. \& Nelkin, B. D. IFI16 is an essential mediator of growth inhibition, but not differentiation, induced by the leukemia inhibitory factor/JAK/STAT pathway in medullary thyroid carcinoma cells. J. Biol. Chem. 280, 4913-4920 (2005).

10. Jin, Y., Wei, Y., Xiong, L., Yang, Y. \& Wu, J. R. Differential regulation of survivin by p53 contributes to cell cycle dependent apoptosis. Cell Res. 15, 361-370 (2005).

11. Rufini, A., Tucci, P., Celardo, I. \& Melino, G. Senescence and aging: the critical roles of p53. Oncogene 32, 5129-5143 (2013).

12. Fu, X., Wu, S., Li, B., Xu, Y. \& Liu, J. Functions of p53 in pluripotent stem cells. Protein Cell 11, 71-78 (2020).

13. Qin, $H$. et al. Regulation of apoptosis and differentiation by $\mathrm{p} 53$ in human embryonic stem cells. J. Biol. Chem. 282, 5842-5852 (2007).

14. Lin, T. et al. p53 induces differentiation of mouse embryonic stem cells by suppressing Nanog expression. Nat. Cell Biol. 7, 165-171 (2005).

15. Zhang, Z. N., Chung, S. K., Xu, Z. \& Xu, Y. Oct4 maintains the pluripotency of human embryonic stem cells by inactivating p53 through Sirt1-mediated deacetylation. Stem Cells 32, 157-165 (2014).

16. Wang, Q. et al. The p53 family coordinates Wnt and nodal inputs in mesendodermal differentiation of embryonic stem cells. Cell Stem Cell 20, 70-86 (2017).

17. Jain, A. K. et al. LnCPRESS1 is a p53-regulated LnCRNA that safeguards pluripotency by disrupting SIRT6-mediated de-acetylation of histone H3K56. Mol. Cell 64, 967-981 (2016).

18. Pappas, K. et al. p53 maintains baseline expression of multiple tumor suppressor genes. Mol. Cancer Res. 15, 1051-1062 (2017).

19. Hafner, A. et al. p53 pulses lead to distinct patterns of gene expression albeit similar DNA-binding dynamics. Nat. Struct. Mol. Biol. 24, 840-847 (2017).

20. Liao, J. C. et al. Interferon-inducible protein 16: insight into the interaction with tumor suppressor p53. Structure 19, 418-429 (2011).

21. Harding, A. et al. Highly efficient differentiation of endothelial cells from pluripotent stem cells requires the MAPK and the PI3K pathways. Stem Cells 35, 909-919 (2017).

22. Rahman, F. et al. Ascorbic acid drives the differentiation of mesoderm-derived embryonic stem cells. Involvement of p38 MAPK/CREB and SVCT2 transporter. Mol. Nutr. Food Res. 61, 1600506 (2017).

23. Hamilton, W. B. \& Brickman, J. M. Erk signaling suppresses embryonic stem cell self-renewal to specify endoderm. Cell Rep. 9, 2056-2070 (2014).

24. Clarke, C. J. et al. Critical role of the transcription factor AP-1 for the constitutive and interferon-induced expression of IFI 16. J. Cell Biochem. 89, 80-93 (2003)

25. Sinner, D., Rankin, S., Lee, M. \& Zorn, A. M. Sox17 and beta-catenin cooperate to regulate the transcription of endodermal genes. Development 131, 3069-3080 (2004).

26. Xu, X. et al. Endoderm and pancreatic islet lineage differentiation from human embryonic stem cells. Cloning Stem Cells 8, 96-107 (2006).

27. D'Amour, K. A. et al. Efficient differentiation of human embryonic stem cells to definitive endoderm. Nat. Biotechnol. 23, 1534-1541 (2005).

28. Technau, U. Brachyury, the blastopore and the evolution of the mesoderm. Bioessays 23, 788-794 (2001)

29. Nelson, T. J. et al. CXCR4+/FLK-1+ biomarkers select a cardiopoietic lineage from embryonic stem cells. Stem Cells 26, 1464-1473 (2008).

30. Nishina, S. et al. PAX6 expression in the developing human eye. Br. J. Ophthalmol. 83, 723-727 (1999).

31. Mortensen, A. H., Schade, V., Lamonerie, T. \& Camper, S. A. Deletion of OTX2 in neural ectoderm delays anterior pituitary development. Hum. Mol. Genet. 24, 939-953 (2015).

32. Lendahl, U., Zimmerman, L. B. \& McKay, R. D. CNS stem cells express a new class of intermediate filament protein. Cell 60, 585-595 (1990).

33. Oram, K. F., Carver, E. A. \& Gridley, T. Slug expression during organogenesis in mice. Anat. Rec. A Disco. Mol. Cell Evol. Biol. 271, 189-191 (2003).

34. Kenzelmann Broz, D. et al. Global genomic profiling reveals an extensive p53regulated autophagy program contributing to key p53 responses. Genes Dev. 27, 1016-1031 (2013)

35. Alexander, R. P., Fang, G., Rozowsky, J., Snyder, M. \& Gerstein, M. B. Annotating non-coding regions of the genome. Nat. Rev. Genet. 11, 559-571 (2010).

36. Halfon, M. S. Studying transcriptional enhancers: the founder fallacy, validation creep, and other biases. Trends Genet. 35, 93-103 (2019).

37. Kim, H. S. et al. Pluripotency factors functionally premark cell-type-restricted enhancers in ES cells. Nature 556, 510-514 (2018).
38. Krijger, P. H. \& de Laat, W. Regulation of disease-associated gene expression in the 3D genome. Nat. Rev. Mol. Cell Biol. 17, 771-782 (2016).

39. Kamada, R., Toguchi, Y., Nomura, T., Imagawa, T. \& Sakaguchi, K. Tetramer formation of tumor suppressor protein p53: Structure, function, and applications. Biopolymers 106, 598-612 (2016).

40. Li, X. et al. Long-read ChIA-PET for base-pair-resolution mapping of haplotypespecific chromatin interactions. Nat. Protoc. 12, 899-915 (2017).

41. Mumbach, M. R. et al. HiChIP: efficient and sensitive analysis of protein-directed genome architecture. Nat. Methods 13, 919-922 (2016).

42. Almine, J. F. et al. IFI16 and CGAS cooperate in the activation of STING during DNA sensing in human keratinocytes. Nat. Commun. 8, 14392 (2017).

43. Caposio, P. et al. A novel role of the interferon-inducible protein IFI16 as inducer of proinflammatory molecules in endothelial cells. J. Biol. Chem. 282, 33515-33529 (2007)

\section{ACKNOWLEDGEMENTS}

We thank X. Yu for imaging. This work was supported by the National Natural Science Foundation of China (No. 31900619, 81670702, and 81700683), the Natural Science Foundation of Guangdong (No. 2017 A030310646 and 2018A030310039), China Postdoctoral Science Foundation (No. 2018M631047), and the Science and Technology Project of Shenzhen (No. JCYJ20170307100154602 and GJHZ20180413181702008).

\section{AUTHOR CONTRIBUTIONS}

Q.H. performed almost all the experiments and wrote the paper. Z.B.W. and D.K.J. did hESCs culture and western blots work. W.Y. and C.F.H. did ChIP-seq and RNA-seq analysis. F.R.L., X.F.Y., and N.L. supervised the study and wrote the paper.

\section{COMPETING INTERESTS}

The authors declare no competing interests.

\section{ADDITIONAL INFORMATION}

Supplementary information is available for this paper at https://doi.org/10.1038/ s41536-020-00104-0.

Correspondence and requests for materials should be addressed to X.Y., N.L. or F.L.

Reprints and permission information is available at http://www.nature.com/ reprints

Publisher's note Springer Nature remains neutral with regard to jurisdictional claims in published maps and institutional affiliations.

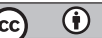

Open Access This article is licensed under a Creative Commons Attribution 4.0 International License, which permits use, sharing, adaptation, distribution and reproduction in any medium or format, as long as you give appropriate credit to the original author(s) and the source, provide a link to the Creative Commons license, and indicate if changes were made. The images or other third party material in this article are included in the article's Creative Commons license, unless indicated otherwise in a credit line to the material. If material is not included in the article's Creative Commons license and your intended use is not permitted by statutory regulation or exceeds the permitted use, you will need to obtain permission directly from the copyright holder. To view a copy of this license, visit http://creativecommons. org/licenses/by/4.0/.

(c) The Author(s) 2020 